\title{
UCRL-TR-215151
}

LAWRENCE LIVERMORE N A TIO NAL LABORATORY

ENDL Ti and $\mathrm{V}$ evaluation updates

David Brown, Rob Hoffman, Kevin Kelley, Bret Beck

September 8, 2005 
This document was prepared as an account of work sponsored by an agency of the United States Government. Neither the United States Government nor the University of California nor any of their employees, makes any warranty, express or implied, or assumes any legal liability or responsibility for the accuracy, completeness, or usefulness of any information, apparatus, product, or process disclosed, or represents that its use would not infringe privately owned rights. Reference herein to any specific commercial product, process, or service by trade name, trademark, manufacturer, or otherwise, does not necessarily constitute or imply its endorsement, recommendation, or favoring by the United States Government or the University of California. The views and opinions of authors expressed herein do not necessarily state or reflect those of the United States Government or the University of California, and shall not be used for advertising or product endorsement purposes.

This work was performed under the auspices of the U.S. Department of Energy by University of California, Lawrence Livermore National Laboratory under Contract W-7405-Eng-48. 


\section{ENDL Ti and V evaluation updates}

\section{David Brown, Rob Hoffman, Kevin Kelley and Bret Beck}

\section{Introduction:}

We need "full" evaluations for the isotopes of Ti and V found naturally. We mean "full" in the sense that we wish to have data suitable for transporting neutrons through the material. In particular, we focus on:

- $\sigma(E)$ for largest cross sections (n,elas), (n,n'), (n,2n), etc.

- $\mathrm{d} \sigma(\mathrm{E}) / \mathrm{d} \Omega$ for $(\mathrm{n}, \mathrm{elas}),\left(\mathrm{n}, \mathrm{n}^{\prime}[\mathrm{i}]\right)$

For the data to be useful, we want the cross section sets and outgoing particle distributions to form a self-consistent set and to have good agreement with experimental data where possible. Also, if possible, we wish:

- $P\left(E, E^{\prime}\right)$ for all outgoing particles

- $\mathrm{P}(\mathrm{E}, \mu)$ for all outgoing particles

- Adequate resonance region

In this note, we will summarize existing evaluations for $\mathrm{Ti}$ and $\mathrm{V}$ and present several strategies for updating all $\mathrm{Ti}$ and $\mathrm{V}$ isotopes for immediate insertion in ENDL and for eventual submission to ENDF/B-VII. We adopt one of these strategies and describe where the data is posted for customer use.

\begin{tabular}{|c|c|c|c|c|c|c|c|c|}
\hline \multicolumn{3}{|c|}{ Isotope } & \multicolumn{6}{|c|}{ Neutron Incident Data (yi01) } \\
\hline & $\mathrm{ZA}$ & Abundance (\%) & HK Data (xs only) & ENDL99 & ENDF/B-VI.r8 & ENDF/B-VII. $\beta 0$ & JENDL3.3 & JEFF3.1 \\
\hline \multirow{8}{*}{$\mathrm{Ti}$} & $\begin{array}{l}\text { za022000 } \\
\text { za022044 } \\
\text { za022045 }\end{array}$ & & $\begin{array}{l}X(2005) \\
X(2005)\end{array}$ & & $\overline{X X(1977,2000)}$ & $\overline{X X(1977,2000)}$ & & \\
\hline & za022046 & 8.25 & $X(2005)$ & & $X(1977)$ & $X(1977)$ & $X(1988,2001)$ & $X(2004)$ \\
\hline & za022047 & 7.44 & $x(2005)$ & & X (1977) & $\times(1977)$ & $X(1988,2001)$ & $x(2004)$ \\
\hline & za022048 & 73.72 & $X(2005)$ & & $X(1977)$ & $X(1977)$ & $X(1988,2001)$ & $X(2004)$ \\
\hline & za022049 & 5.41 & $x(2005)$ & & & & $X(1988,2001)$ & $x(2004)$ \\
\hline & za022050 & 5.18 & $x(2005)$ & & X (1979) & $X(1979)$ & $X(1988,2001)$ & $x(2004)$ \\
\hline & za022051 & & $X(2005)$ & & & & & \\
\hline & $\mathrm{za} 022052$ & & $X(2005)$ & & & & & \\
\hline \multirow{8}{*}{$\mathrm{v}$} & $\mathrm{za} 023000$ & & & & $X(1988)$ & $X(1988)$ & $X(1988,2001)$ & $X(1991)$ \\
\hline & za023046 & & $x(2005)$ & & & & & \\
\hline & za023046m1 & & $x(2005)$ & & & & & \\
\hline & $\mathrm{za} 023047$ & & $\begin{array}{l}X(2005) \\
X(2005)\end{array}$ & & & & & \\
\hline & $\begin{array}{l}\text { za023048 } \\
\text { za023049 }\end{array}$ & & $\begin{array}{l}X(2005) \\
X(2005)\end{array}$ & & & & & \\
\hline & za023050 & 0.25 & $x(2005)$ & & & & & \\
\hline & za023051 & 99.75 & $x(2005)$ & $\mathrm{x}$ & & & & \\
\hline & za023052 & & $X(2005)$ & & & & & \\
\hline
\end{tabular}

Table 1: Table of all available evaluations, with naturally occurring isotopes highlighted in grey. If known, the year of each evaluation is also shown. 


\section{Status of Current Ti Evaluations:}

\section{${ }^{46-48} \mathrm{Ti}$ in ENDF/B-VI, VII}

These evaluations date from the late 1970's (1977) and do not come with very much documentation. The data sets are most likely not internally consistent, the evaluators did not have access to more recent data or models. We will not consider these evaluations further.

\section{${ }^{50} \mathrm{Ti}$ in ENDF/B-VI, VII}

This evaluation dates from the late 1970's (1979) and do not come with very much documentation. The data sets are most likely not internally consistent, the evaluators did not have access to more recent data or models. We will not consider these evaluations further.

\section{${ }^{\text {nat }} \mathrm{Ti}$ in ENDF/B-VI, VII}

This evaluation dates from the late 1970's (1977), and one dataset was updated in 2000. It does not come with very much documentation. The data sets are most likely not internally consistent, the evaluators did not have access to more recent data or models. We will not consider these evaluations further.

\section{6-50 $\mathrm{Ti}$ in JENDL3.3}

While each evaluation was done separately, they were all done as part of the same set of evaluations, first in 1988, then revised in 2001. The cross sections were computed with a mixture of models, but with the same set of optical models. It is not clear whether the other input parameters were held fixed. The outgoing particle distributions were created from a second set of calculations in the 2001 revision. The distributions were all created within the same calculation, so they are internally consistent even if they are not consistent with the cross sections. The resonance region in each isotope were generated separately and are of unknown quality.

\section{${ }^{46-50} \mathrm{Ti}$ in JEFF-3.1}

(n,tot) is fit to nat $\mathrm{Ti}(\mathrm{n}, \mathrm{tot})$, then reused for all isotopes, as is. Means that the natural evaluation is the only one with correct $(n, t o t)$. Similarly for the resonance region. All outgoing distributions are from the same TALYS calculation. Thus, the distributions from these isotopes are equivalent to what we will get from TALYS, running in the same (default) mode. The TALYS calculations were used as an uninformative prior for a generalized least-square fit to all available cross section data. The data itself was also evaluated and covariance assigned. Thus, the cross-section fits are most likely the highest quality possible. Nevertheless, given that the $(n, t o t)$ is fit to the natural data, the sub-cross sections cannot add up to the total, so unitarity is not conserved here and the cross section data is probably inappropriate for transport. Furthermore, in the translation 
of this data from ENDF/B format to ENDL format, we uncovered several bugs that further render this data useless. First, the outgoing gamma spectrum from the decay out of the continuum for $(n, \gamma)$ reactions has all of the outgoing energies set to $0 \mathrm{MeV}$. This bug means that energy cannot be conserved for this reaction. Second, the Kalbach data for outgoing distributions produces probability distributions that are not quite normalized to unity. Finally, the energy grids for several of the cross section files are not synchronized with the corresponding outgoing particle probability distributions. This would be $\mathrm{OK}$, if the files at least had the same threshold energies. Unfortunately, they do not. We will not consider these evaluations further.

\section{${ }^{44-52} \mathrm{Ti}$ from R. Hoffman and K. Kelley}

Self consistent set of STAPRE calculations across entire mass range. Not necessarily best cross section set for a specific isotope, but likely best overall. Used KoningDelaroche. Cross section data only so suitable for radiochemistry, but not complete enough for transport problems.

\section{Status of Current V Evaluations:}

Given the natural isotopic abundance of this isotope $\left(99.75 \%{ }^{51} \mathrm{~V}\right)$, the natural evaluations can be safely compared with the ${ }^{51} \mathrm{~V}$ evaluations from Hoffman and Kelley and from ENDL99.

\section{${ }^{\text {nat }} \mathbf{V}$ in ENDF/B-VI, VII}

Evaluation documented in A.B. Smith, D.L. Smith, P.T. Guenther et al., Argonne National Laboratory Report ANL/NDM-105 (1988). Only $\gamma$ spectrum from capture was updated in 2000. The cross sections were modeled using a set of Hauser-Feshbach calculations, but reproduce the data well. Only inelastic outgoing neutron distributions are given for the particle out channels.

\section{${ }^{\text {nat }} \mathbf{V}$ in JENDL3.3}

This evaluation was performed in 1988, then revised in 2001. The revision consisted of updating the outgoing particle distributions and the resonance region. The cross sections were computed with a mixture of models, but with the same set of optical models. It is not clear whether the other input parameters were held fixed. The distributions were all created within the same calculation, so they are internally consistent even if they are not consistent with the cross sections.

\section{${ }^{\text {nat}} \mathbf{V}$ in JEFF-3.1}

This evaluation is not very well documented. What we can tell from the evaluation documentation is that the outgoing particle distributions were all computed within the same set of IDA calculations. Given the evaluators (M. Herman, F. Fabbri, G. Reffo), we suspect that the cross section files were computed within STAPRE, but this is not clear. The evaluation was adopted from the EFF-3.0 file. 


\section{${ }^{51} \mathrm{~V}$ from ENDL99}

No documentation is given, so we do not consider this evaluation further.

\section{${ }^{46-53}$ V from R. Hoffman and K. Kelley}

Self consistent set of STAPRE calculations across entire mass range. Not necessarily best cross section set for a specific isotope, but likely best overall. Used KoningDelaroche. . Cross section data only so suitable for radiochemistry, but not complete enough for transport problems.

\section{Updating ENDL99:}

As part of our updating, there are several approaches we could take. In all cases, we have to define the scope of isotopes we must include. There are 3 options here: 1) natural evaluation only 2) naturally occurring isotopes only 3 ) all radiochemically important isotopes. Whichever scope is adopted, we must choose what strategy to use to produce the updated sets. Here the options are:

1. Steal JEFF3.1/JENDL3.3 evaluation set

a. PROS:

i. Fast, just need to work out fete bugs

b. CONS:

i. None of the evaluated data is internally consistent

ii. Only gets data for stable isotopes

2. Run TALYS or EMPIRE, steal JEFF3.1/JENDL3.3 resonance region

a. PROS:

i. All evaluations are internally consistent by construction

ii. Gets all isotopes considered by Hoffman and Kelley

iii. Faster than options \#3, \#4

b. CONS:

i. Slower than options \#1(must repackage TALYS output)

ii. Cross sections not highest possible quality

iii. Matching onto resonance regions must be done with care

3. Run TALYS or EMPIRE, replace cross sections with those from R. Hoffman and K. Kelley, steal JEFF3.1/JENDL3.3 resonance region

a. PROS:

i. All cross sections will be internally consistent

ii. Gets all isotopes considered by Hoffman and Kelley

iii. Faster than option \#4

b. CONS:

i. Slower than options \#1,\#2 (must repackage TALYS output)

ii. None of the outgoing particle distributions will be consistent with the cross sections

iii. Cross sections not highest possible quality 
iv. Matching onto resonance regions must be done with care

4. Run TALYS or EMPIRE, using parameters tuned to reproduce R. Hoffman and K. Kelley, steal JEFF3.1/JENDL3.3 resonance region calculations

a. PROS:

i. All of evaluations can be made internally consistent

b. CONS:

ii. Gets all isotopes considered by Hoffman and Kelley

i. Time consuming, must convert STAPRE inputs into TALYS format and TALYS output into ENDL

ii. Matching onto resonance regions must be done with care

We will adopt option \#1 to produce the required library additions. For the titanium isotopes, we will choose the JENDL3.3 evaluations. The cross section evaluated data is internally consistent and is in good agreement with data, where available. Furthermore, the outgoing particle distributions appear to be complete. For vanadium, the situation is a little trickier. Here, there is only natural evaluations (with the exception of ENDL99), but endep cannot currently compute energy and momentum depositions for natural targets. As a stopgap, we will compute the energy and momentum depositions on ENDL99' ${ }^{51} \mathrm{~V}$ evaluation, then copy them into JENDL3.3's natV evaluation. This is not as bad a mistake as it seems $-{ }^{\text {nat }} \mathrm{V}$ is $95.75 \%{ }^{51} \mathrm{~V}$ and both ENDL99 and JENDL3.3's cross section data are in reasonable agreement.

\section{Evaluated and Processed data availability on OCF/SCF:}

The data will be mirrored on the SCF and OCF with the same directory structure. The evaluated data will be stored in: /usr/gapps/data/nuclear/evaluated/TI_V/

The processed data will be stored in two places. For Monte-Carlo transport, the processed data will be stored in: /usr/gapps/data/nuclear/processed/betas/end199_Ti_V/mcf/ For deterministic transport, the data will be in: /usr/gapps/data/nuclear/processed/betas/end199_Ti_V/ndf/

\section{Contents of the JENDL3.3 Evaluations:}

See attached spreadsheet.

\section{Comparison of cross section data with experiment:}

See attached plots. 


\begin{tabular}{|c|c|c|c|c|c|c|c|c|c|}
\hline za & Reaction & C & $\sigma(E)$ & $\begin{array}{l}\text { Outgoing } \\
\text { particles }\end{array}$ & $P(E, \mu)$ & $\mathrm{P}\left(\mathrm{E}, \mu, \mathrm{E}^{\prime}\right)$ & $M\left(E, E^{\prime}\right)$ & $<E^{\prime}>(E)$ & $<p>(E)$ \\
\hline \multirow[t]{32}{*}{ za022046 } & (yi,tot) & 1 & $\bar{Y}$ & & & & & & \\
\hline & (yi,elas) & 10 & $\bar{Y}$ & & & & & $\bar{Y}$ & $\bar{Y}$ \\
\hline & & & & $\mathrm{n}$ & $Y$ & & & $Y$ & $Y$ \\
\hline & $\overline{(y i, n)}$ & 11 & $\bar{Y}$ & & & & & $\bar{Y}$ & $\bar{Y}$ \\
\hline & & & & $\mathrm{n}$ & & $Y$ & & $Y$ & $Y$ \\
\hline & & & & $g$ & & $Y$ & $Y$ & $Y$ & $Y$ \\
\hline & $(\mathrm{yi}, \mathrm{n}[\mathrm{i}])$ & 11 & $\mathrm{Y}$ & & & & & $\mathrm{Y}$ & $\mathrm{Y}$ \\
\hline & & & & $\mathrm{n}$ & $Y$ & & & $Y$ & $Y$ \\
\hline & & & & $g$ & & $Y$ & $Y$ & $Y$ & $Y$ \\
\hline & $\overline{(y i, 2 n)}$ & 12 & $\bar{Y}$ & & & & & $\bar{Y}$ & $\bar{Y}$ \\
\hline & & & & $\mathrm{n}$ & & $Y$ & & $Y$ & $Y$ \\
\hline & & & & $\mathrm{g}$ & & $Y$ & $Y$ & $Y$ & $Y$ \\
\hline & $(y i, 2 p)$ & 18 & $\mathrm{Y}$ & & & & & $Y$ & $Y$ \\
\hline & $(y i, n p)$ & 20 & $\bar{Y}$ & & & & & $\bar{Y}$ & $\bar{Y}$ \\
\hline & & & & $\mathrm{n}$ & & $Y$ & & $Y$ & $Y$ \\
\hline & & & & $g$ & & $Y$ & $Y$ & $Y$ & $Y$ \\
\hline & $(y i, n a)$ & 26 & $\mathrm{Y}$ & & & & & $\mathrm{Y}$ & $\mathrm{Y}$ \\
\hline & & & & $\mathrm{n}$ & & $Y$ & & $Y$ & $Y$ \\
\hline & & & & $g$ & & $Y$ & $Y$ & $Y$ & $Y$ \\
\hline & $\overline{(y i, p)}$ & 40 & $\mathrm{Y}$ & & & & & $\bar{Y}$ & $\bar{Y}$ \\
\hline & & & & $\mathrm{g}$ & & $Y$ & $Y$ & $Y$ & $Y$ \\
\hline & $(y i, d)$ & 41 & $\mathrm{Y}$ & & & & & $\mathrm{Y}$ & $\mathrm{Y}$ \\
\hline & & & & $\mathrm{g}$ & & $Y$ & $Y$ & $Y$ & $Y$ \\
\hline & $(y i, t)$ & 42 & $\mathrm{Y}$ & & & & & $Y$ & $Y$ \\
\hline & $(\mathrm{yi}, \mathrm{He} 3)$ & 44 & $\mathrm{Y}$ & & & & & $Y$ & $Y$ \\
\hline & $(y i, a)$ & 45 & $\mathrm{Y}$ & & & & & $\mathrm{Y}$ & $\mathrm{Y}$ \\
\hline & & & & $g$ & & $Y$ & $Y$ & $Y$ & $Y$ \\
\hline & $\overline{(y i, g)}$ & 46 & $\mathrm{Y}$ & & & & & $Y$ & $\mathrm{Y}$ \\
\hline & & & & $\mathrm{g}$ & & $Y$ & $Y$ & $Y$ & $Y$ \\
\hline & $(y i, x p)$ & 50 & $\mathrm{Y}$ & & & & & & \\
\hline & & & & p & $Y$ & $Y$ & & & \\
\hline & $(y i, X a)$ & 54 & $\mathrm{Y}$ & & & & & & \\
\hline
\end{tabular}




\begin{tabular}{|c|c|c|c|c|c|c|c|c|c|}
\hline $\mathrm{za}$ & Reaction & C & $\sigma(E)$ & $\begin{array}{l}\text { Outgoing } \\
\text { particles }\end{array}$ & $P(E, \mu)$ & $P\left(E, \mu, E^{\prime}\right)$ & $M\left(E, E^{\prime}\right)$ & $\left\langle\mathrm{E}^{\prime}\right\rangle(\mathrm{E})$ & $<p>(E)$ \\
\hline \multirow[t]{32}{*}{ za022047 } & (yi,tot) & 1 & $\bar{Y}$ & & & & & & \\
\hline & (yi,elas) & 10 & $\mathrm{Y}$ & & & & & $Y$ & $Y$ \\
\hline & & & & $\mathrm{n}$ & $Y$ & & & $Y$ & $Y$ \\
\hline & $\overline{(y i, n)}$ & 11 & $\mathrm{Y}$ & & & & & $Y$ & $Y$ \\
\hline & & & & $\mathrm{n}$ & & Y & & $Y$ & $Y$ \\
\hline & & & & $\mathrm{g}$ & & $Y$ & $Y$ & $Y$ & $Y$ \\
\hline & $(y i, n[i])$ & 11 & $\bar{Y}$ & & & & & $\mathrm{Y}$ & $\mathrm{Y}$ \\
\hline & & & & $\mathrm{n}$ & $Y$ & & & $Y$ & $Y$ \\
\hline & & & & $\mathrm{g}$ & & $Y$ & $Y$ & $Y$ & $Y$ \\
\hline & $(y i, 2 n)$ & 12 & $\bar{Y}$ & & & & & $\mathrm{Y}$ & $\mathrm{Y}$ \\
\hline & & & & $n$ & & $Y$ & & $Y$ & $Y$ \\
\hline & & & & $\mathrm{g}$ & & $Y$ & $Y$ & $Y$ & $Y$ \\
\hline & (yi,2p) & 18 & $Y$ & & & & & $Y$ & $\mathrm{Y}$ \\
\hline & $(y i, n$ p) & 20 & $\mathrm{Y}$ & & & & & $\mathrm{Y}$ & $\mathrm{Y}$ \\
\hline & & & & $n$ & & $Y$ & & $Y$ & $Y$ \\
\hline & & & & $\mathrm{g}$ & & $Y$ & $Y$ & $Y$ & $Y$ \\
\hline & $(y i, n a)$ & 26 & $\bar{Y}$ & & & & & $Y$ & $Y$ \\
\hline & & & & $n$ & & Y & & $Y$ & $Y$ \\
\hline & & & & $\mathrm{g}$ & & $Y$ & $Y$ & $Y$ & $Y$ \\
\hline & $(y i, p)$ & 40 & $\bar{Y}$ & & & & & $Y$ & $\mathrm{Y}$ \\
\hline & & & & $\mathrm{g}$ & & $Y$ & $Y$ & $Y$ & $Y$ \\
\hline & $(y i, d)$ & 41 & $\bar{Y}$ & & & & & $Y$ & $\mathrm{Y}$ \\
\hline & & & & $\mathrm{g}$ & & $Y$ & $Y$ & $Y$ & $Y$ \\
\hline & $(y i, t)$ & 42 & $\mathrm{Y}$ & & & & & $Y$ & $Y$ \\
\hline & $(\mathrm{yi}, \mathrm{He} 3)$ & 44 & $\bar{Y}$ & & & & & $\mathrm{Y}$ & $\mathrm{Y}$ \\
\hline & $(y i, a)$ & 45 & $Y$ & & & & & $\mathrm{Y}$ & $\mathrm{Y}$ \\
\hline & & & & $\mathrm{g}$ & & $Y$ & $Y$ & $Y$ & $Y$ \\
\hline & $(y i, g)$ & 46 & $\mathrm{Y}$ & & & & & $Y$ & $Y$ \\
\hline & & & & $\mathrm{g}$ & & Y & $Y$ & $Y$ & $Y$ \\
\hline & $(y i, x p)$ & 50 & $\bar{Y}$ & & & & & & \\
\hline & & & & $p$ & $Y$ & $Y$ & & & \\
\hline & $(y i, x a)$ & 54 & $\mathrm{Y}$ & $a$ & $Y$ & $Y$ & & & \\
\hline
\end{tabular}




\begin{tabular}{|c|c|c|c|c|c|c|c|c|c|}
\hline $\mathrm{za}$ & Reaction & c & $\sigma(\mathrm{E})$ & $\begin{array}{l}\text { Outgoing } \\
\text { particles } \\
\end{array}$ & $\mathrm{P}(\mathrm{E}, \mu)$ & $\mathrm{P}\left(\mathrm{E}, \mu, \mathrm{E}^{\prime}\right)$ & $M\left(E, E^{\prime}\right)$ & $<E^{\prime}>(E)$ & $<\mathrm{p}>(\mathrm{E})$ \\
\hline \multirow[t]{31}{*}{ za022048 } & (yi,tot) & 1 & $\mathrm{Y}$ & & & & & & \\
\hline & (yi,elas) & 10 & $\mathrm{Y}$ & & & & & $Y$ & $\mathrm{Y}$ \\
\hline & & & & $\mathrm{n}$ & $Y$ & & & $Y$ & Y \\
\hline & $(y i, n)$ & 11 & $\mathrm{Y}$ & & & & & $\mathrm{Y}$ & $\mathrm{Y}$ \\
\hline & & & & $\mathrm{n}$ & & $Y$ & & $Y$ & Y \\
\hline & & & & g & & $Y$ & $Y$ & $Y$ & Y \\
\hline & $(y i, n[i])$ & 11 & $\mathrm{Y}$ & & & & & $\mathrm{Y}$ & $\mathrm{Y}$ \\
\hline & & & & $\mathrm{n}$ & $Y$ & & & $Y$ & Y \\
\hline & & & & $\mathrm{g}$ & & $Y$ & $Y$ & $Y$ & Y \\
\hline & $(y i, 2 n)$ & 12 & $\mathrm{Y}$ & & & & & $Y$ & $\mathrm{Y}$ \\
\hline & & & & $\mathrm{n}$ & & $Y$ & & $\mathrm{Y}$ & Y \\
\hline & & & & $g$ & & $Y$ & $Y$ & $Y$ & Y \\
\hline & $(y i, n p)$ & 20 & $\mathrm{Y}$ & & & & & $\mathrm{Y}$ & $\mathrm{Y}$ \\
\hline & & & & $\mathrm{n}$ & & $Y$ & & Y & $Y$ \\
\hline & & & & $\mathrm{g}$ & & $Y$ & Y & $Y$ & Y \\
\hline & $(y i, n a)$ & 26 & $\mathrm{Y}$ & & & & & $\mathrm{Y}$ & $\mathrm{Y}$ \\
\hline & & & & $\mathrm{n}$ & & $Y$ & & $Y$ & Y \\
\hline & & & & $\mathrm{g}$ & & $Y$ & $Y$ & $Y$ & Y \\
\hline & $(y i, p)$ & 40 & $\mathrm{Y}$ & & & & & $\mathrm{Y}$ & $\mathrm{Y}$ \\
\hline & & & & $\mathrm{g}$ & & $Y$ & $Y$ & $Y$ & Y \\
\hline & $(y i, d)$ & 41 & $\mathrm{Y}$ & & & & & $\mathrm{Y}$ & $\mathrm{Y}$ \\
\hline & & & & $\mathrm{g}$ & & $Y$ & $Y$ & $Y$ & Y \\
\hline & $(y i, t)$ & 42 & $Y$ & & & & & $Y$ & $\mathrm{Y}$ \\
\hline & $(\mathrm{yi}, \mathrm{He} 3)$ & 44 & $Y$ & & & & & $Y$ & $\mathrm{Y}$ \\
\hline & $(y i, a)$ & 45 & $\mathrm{Y}$ & & & & & $\mathrm{Y}$ & $\mathrm{Y}$ \\
\hline & & & & $\mathrm{g}$ & & $Y$ & $Y$ & $Y$ & Y \\
\hline & $(y i, g)$ & 46 & $\mathrm{Y}$ & & & & & $Y$ & $\mathrm{Y}$ \\
\hline & & & & $\mathrm{g}$ & & $Y$ & $Y$ & $Y$ & Y \\
\hline & $(y i, x p)$ & 50 & Y & & & & & & \\
\hline & & & & $\mathrm{p}$ & $Y$ & $Y$ & & & \\
\hline & $(y i, x \quad a)$ & 54 & $\mathrm{Y}$ & & y & v & & & \\
\hline
\end{tabular}




\begin{tabular}{|c|c|c|c|c|c|c|c|c|c|}
\hline za & Reaction & C & $\sigma(\mathrm{E})$ & $\begin{array}{l}\text { Outgoing } \\
\text { particles }\end{array}$ & $P(E, \mu)$ & $\mathrm{P}\left(\mathrm{E}, \mu, \mathrm{E}^{\prime}\right)$ & $M\left(E, E^{\prime}\right)$ & $<E^{\prime}>(E)$ & $<p>(E)$ \\
\hline \multirow[t]{32}{*}{ za022049 } & (yi,tot) & 1 & $\bar{Y}$ & & & & & & \\
\hline & (yi,elas) & 10 & $\mathrm{Y}$ & & & & & $\mathrm{Y}$ & $\begin{array}{c}\mathrm{Y} \\
\end{array}$ \\
\hline & & & & $\mathrm{n}$ & $Y$ & & & $Y$ & Y \\
\hline & $(y i, n)$ & 11 & $\mathrm{Y}$ & & & & & $\mathrm{Y}$ & $\begin{array}{l}\mathrm{Y} \\
\end{array}$ \\
\hline & & & & $\mathrm{n}$ & & $Y$ & & $Y$ & Y \\
\hline & & & & $g$ & & $Y$ & $Y$ & $Y$ & Y \\
\hline & $(\mathrm{yi}, \mathrm{n}[\mathrm{i}])$ & 11 & $\mathrm{Y}$ & & & & & $\mathrm{Y}$ & $\mathrm{Y}$ \\
\hline & & & & $\mathrm{n}$ & $Y$ & & & $Y$ & Y \\
\hline & & & & $\mathrm{g}$ & & $Y$ & $Y$ & $Y$ & Y \\
\hline & $(y i, 2 n)$ & 12 & $\mathrm{Y}$ & & & & & $\mathrm{Y}$ & $\mathrm{Y}$ \\
\hline & & & & $\mathrm{n}$ & & $Y$ & & $Y$ & Y \\
\hline & & & & $\mathrm{g}$ & & $Y$ & $Y$ & $Y$ & Y \\
\hline & $(y i, 2 p)$ & 18 & $Y$ & & & & & $Y$ & $\mathrm{Y}$ \\
\hline & $(y i, n p)$ & 20 & $\mathrm{Y}$ & & & & & $\mathrm{Y}$ & $\begin{array}{l}\mathrm{Y} \\
\mathrm{y}\end{array}$ \\
\hline & & & & $\mathrm{n}$ & & $Y$ & & $Y$ & Y \\
\hline & & & & $\mathrm{g}$ & & $Y$ & $Y$ & $Y$ & Y \\
\hline & $(y i, n a)$ & 26 & $\mathrm{Y}$ & & & & & $\mathrm{Y}$ & $\mathrm{Y}$ \\
\hline & & & & $\mathrm{n}$ & & $Y$ & & $Y$ & Y \\
\hline & & & & $\mathrm{g}$ & & $Y$ & $Y$ & $Y$ & Y \\
\hline & $(y i, p)$ & 40 & $\mathrm{Y}$ & & & & & $Y$ & $\mathrm{Y}$ \\
\hline & & & & $\mathrm{g}$ & & $Y$ & $Y$ & $Y$ & Y \\
\hline & $(y i, d)$ & 41 & $\mathrm{Y}$ & & & & & $\mathrm{Y}$ & $\mathrm{Y}$ \\
\hline & & & & $\mathrm{g}$ & & $Y$ & $Y$ & $Y$ & Y \\
\hline & $(y i, t)$ & 42 & $\mathrm{Y}$ & & & & & $Y$ & $\mathrm{Y}$ \\
\hline & $(\mathrm{yi}, \mathrm{He} 3)$ & 44 & $\mathrm{Y}$ & & & & & $Y$ & $\mathrm{Y}$ \\
\hline & $(y i, a)$ & 45 & $\mathrm{Y}$ & & & & & $\mathrm{Y}$ & $\begin{array}{c}\mathrm{Y} \\
\end{array}$ \\
\hline & & & & $\mathrm{g}$ & & $Y$ & $Y$ & $Y$ & Y \\
\hline & $\overline{(y i, g)}$ & 46 & $\mathrm{Y}$ & & & & & $Y$ & $\mathrm{Y}$ \\
\hline & & & & $\mathrm{g}$ & & $Y$ & $Y$ & $Y$ & Y \\
\hline & $(y i, x p)$ & 50 & $\mathrm{Y}$ & & & & & & \\
\hline & & & & p & $Y$ & $Y$ & & & \\
\hline & $(y i, x$ a) & 54 & Y & 7 & $Y$ & y & & & \\
\hline
\end{tabular}




\begin{tabular}{|c|c|c|c|c|c|c|c|c|c|}
\hline $\mathrm{za}$ & Reaction & $\mathrm{C}$ & $\sigma(E)$ & $\begin{array}{l}\text { Outgoing } \\
\text { particles }\end{array}$ & $P(E, \mu)$ & $P\left(E, \mu, E^{\prime}\right)$ & $M\left(E, E^{\prime}\right)$ & $\left\langle\mathrm{E}^{\prime}\right\rangle(\mathrm{E})$ & $<p>(E)$ \\
\hline \multirow[t]{35}{*}{ za022050 } & (yi,tot) & 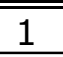 & $\overline{\bar{Y}}$ & & & & & & \\
\hline & (yi,elas) & 10 & $Y$ & & & & & $Y$ & $Y$ \\
\hline & & & & $\mathrm{n}$ & $Y$ & & & $Y$ & $Y$ \\
\hline & $\overline{(y i, n)}$ & 11 & $\bar{Y}$ & & & & & $Y$ & $Y$ \\
\hline & & & & $n$ & & Y & & $Y$ & $Y$ \\
\hline & & & & $\mathrm{g}$ & & $Y$ & $Y$ & $Y$ & $Y$ \\
\hline & $(y i, n[i])$ & 11 & $\bar{Y}$ & & & & & $\mathrm{Y}$ & $\bar{Y}$ \\
\hline & & & & $\mathrm{n}$ & $Y$ & & & $Y$ & $Y$ \\
\hline & & & & $\mathrm{g}$ & & $Y$ & $Y$ & $Y$ & $Y$ \\
\hline & $(y i, 2 n)$ & 12 & Y & & & & & $Y$ & $Y$ \\
\hline & & & & $n$ & & $Y$ & & $Y$ & $Y$ \\
\hline & & & & $\mathrm{g}$ & & $Y$ & $Y$ & $Y$ & $Y$ \\
\hline & $(y i, 3 n)$ & 13 & $\bar{Y}$ & & & & & $Y$ & $\mathrm{Y}$ \\
\hline & & & & $n$ & & $Y$ & & $Y$ & $Y$ \\
\hline & & & & $\mathrm{g}$ & & & & $Y$ & $Y$ \\
\hline & $(y i, n p)$ & 20 & $Y$ & & & & & $Y$ & $Y$ \\
\hline & & & & $n$ & & $Y$ & & $Y$ & $Y$ \\
\hline & & & & $\mathrm{g}$ & & $Y$ & $Y$ & $Y$ & $Y$ \\
\hline & $(y i, n$ a) & 26 & $\mathrm{Y}$ & & & & & $Y$ & $Y$ \\
\hline & & & & $n$ & & $Y$ & & $Y$ & $Y$ \\
\hline & & & & $\mathrm{g}$ & & $Y$ & $Y$ & $Y$ & $Y$ \\
\hline & $\overline{(y i, p)}$ & 40 & $\bar{Y}$ & & & & & $Y$ & $Y$ \\
\hline & & & & $\mathrm{g}$ & & $Y$ & $Y$ & $Y$ & $Y$ \\
\hline & $(y i, d)$ & 41 & $\bar{Y}$ & & & & & $\mathrm{Y}$ & $\bar{Y}$ \\
\hline & & & & $\mathrm{g}$ & & $Y$ & $Y$ & $Y$ & $Y$ \\
\hline & $(y i, t)$ & 42 & $Y$ & & & & & $Y$ & $\mathrm{Y}$ \\
\hline & $(\mathrm{yi}, \mathrm{He} 3)$ & 44 & $Y$ & & & & & $Y$ & $Y$ \\
\hline & $(y i, a)$ & 45 & $Y$ & & & & & $Y$ & $Y$ \\
\hline & & & & $\mathrm{g}$ & & $Y$ & $Y$ & $Y$ & $Y$ \\
\hline & $\overline{(y i, g)}$ & 46 & $\mathrm{Y}$ & & & & & $Y$ & $Y$ \\
\hline & & & & $\mathrm{g}$ & & $Y$ & $Y$ & $Y$ & $Y$ \\
\hline & $(y i, x p)$ & 50 & $Y$ & & & & & & \\
\hline & & & & $p$ & $Y$ & $Y$ & & & \\
\hline & $(y i, x$ a) & 54 & $\bar{Y}$ & & & & & & \\
\hline & & & & a & $Y$ & $Y$ & & & \\
\hline
\end{tabular}




\begin{tabular}{|c|c|c|c|c|c|c|c|c|c|}
\hline $\mathrm{za}$ & Reaction & $\mathrm{C}$ & $\sigma(E)$ & $\begin{array}{l}\text { Outgoing } \\
\text { particles }\end{array}$ & $P(E, \mu)$ & $P\left(E, \mu, E^{\prime}\right)$ & $M\left(E, E^{\prime}\right)$ & $\left.<\mathrm{E}^{\prime}\right\rangle(\mathrm{E})$ & $<p>(E)$ \\
\hline \multirow[t]{35}{*}{ za023000 } & (yi,tot) & 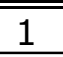 & $\overline{\bar{Y}}$ & & & & & & \\
\hline & (yi,elas) & 10 & $\bar{Y}$ & & & & & & \\
\hline & & & & $\mathrm{n}$ & $Y$ & & & & \\
\hline & $(y i, n)$ & 11 & $\mathrm{Y}$ & & & & & & \\
\hline & & & & $\mathrm{n}$ & & Y & & & \\
\hline & & & & $\mathrm{g}$ & & $Y$ & $Y$ & & \\
\hline & $(y i, n[i])$ & 11 & $\bar{Y}$ & & & & & & \\
\hline & & & & $\mathrm{n}$ & $Y$ & & & & \\
\hline & & & & $\mathrm{g}$ & & $Y$ & $Y$ & & \\
\hline & $(y i, 2 n)$ & 12 & Y & & & & & & \\
\hline & & & & $n$ & & Y & & & \\
\hline & & & & $\mathrm{g}$ & & $Y$ & $Y$ & & \\
\hline & $(y i, n$ p) & 20 & $\bar{Y}$ & & & & & & \\
\hline & & & & $n$ & & Y & & & \\
\hline & & & & $\mathrm{g}$ & & $Y$ & $Y$ & & \\
\hline & $(y i, n a)$ & 26 & $Y$ & & & & & & \\
\hline & & & & $n$ & & $Y$ & & & \\
\hline & & & & $\mathrm{g}$ & & $Y$ & $Y$ & & \\
\hline & $(y i, p)$ & 40 & $\mathrm{Y}$ & & & & & & \\
\hline & & & & $\mathrm{g}$ & & $Y$ & $Y$ & & \\
\hline & $(y i, d)$ & 41 & $\mathrm{Y}$ & & & & & & \\
\hline & & & & $\mathrm{g}$ & & $Y$ & $Y$ & & \\
\hline & $(y i, t)$ & 42 & $\mathrm{Y}$ & & & & & & \\
\hline & & & & $\mathrm{g}$ & & $Y$ & $Y$ & & \\
\hline & $\overline{(y i, a)}$ & 45 & $\bar{Y}$ & & & & & & \\
\hline & & & & $\mathrm{g}$ & & $Y$ & $Y$ & & \\
\hline & $(y i, g)$ & 46 & $Y$ & & & & & & \\
\hline & & & & $\mathrm{g}$ & & $Y$ & $Y$ & & \\
\hline & $(y i, x p)$ & 50 & $\mathrm{Y}$ & & & & & & \\
\hline & & & & $p$ & $Y$ & $Y$ & & & \\
\hline & $(y i, x d)$ & 51 & $Y$ & & & & & & \\
\hline & $(y i, x t)$ & 52 & $Y$ & & & & & & \\
\hline & & & & $\mathrm{t}$ & $Y$ & $Y$ & & & \\
\hline & $(y i, x$ a) & 54 & $\mathrm{Y}$ & & & & & & \\
\hline & & & & a & $Y$ & $Y$ & & & \\
\hline
\end{tabular}


${ }^{46} \mathrm{Ti}_{g}(\mathrm{n}, 2 \mathrm{n}){ }^{45} \mathrm{Ti}$

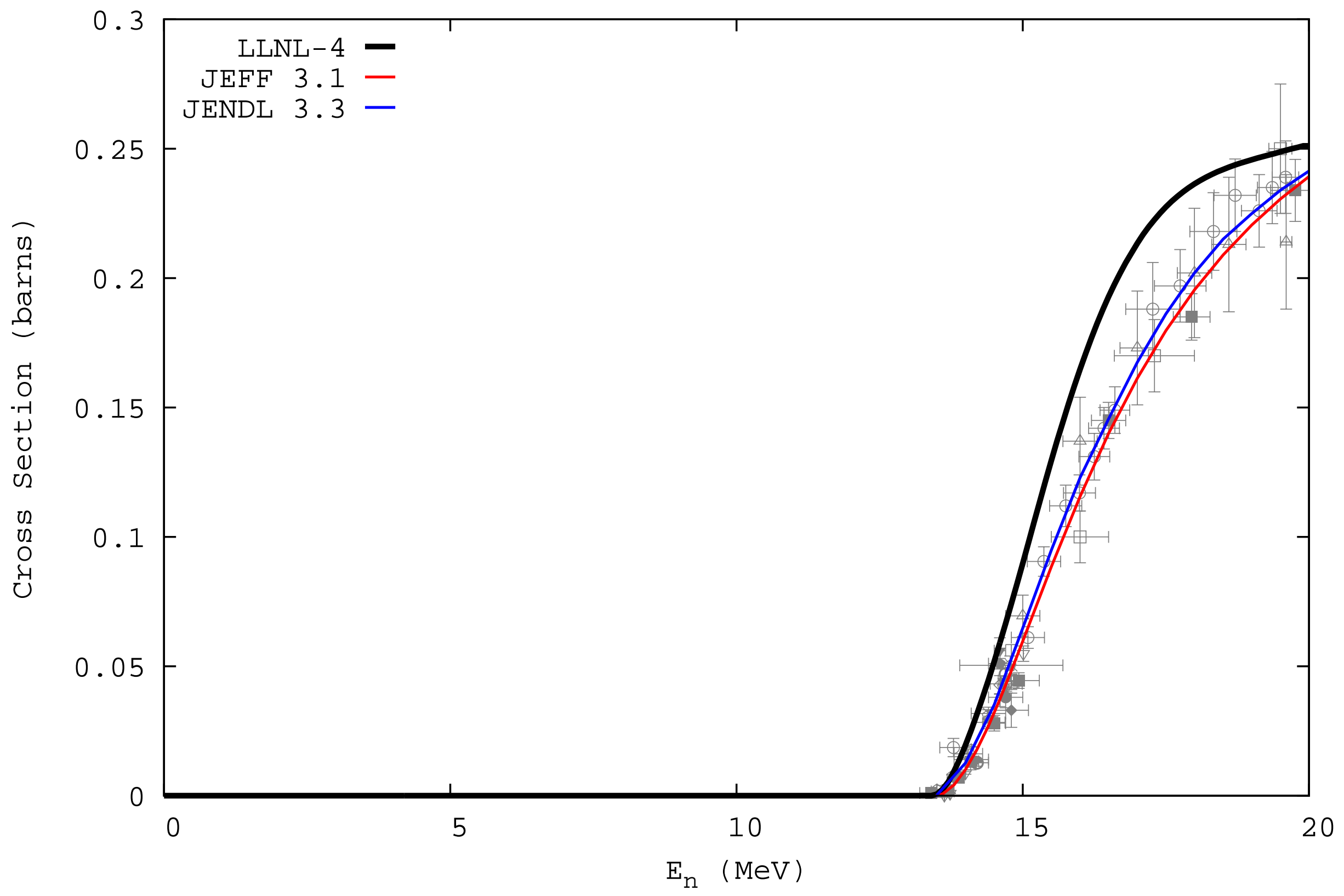




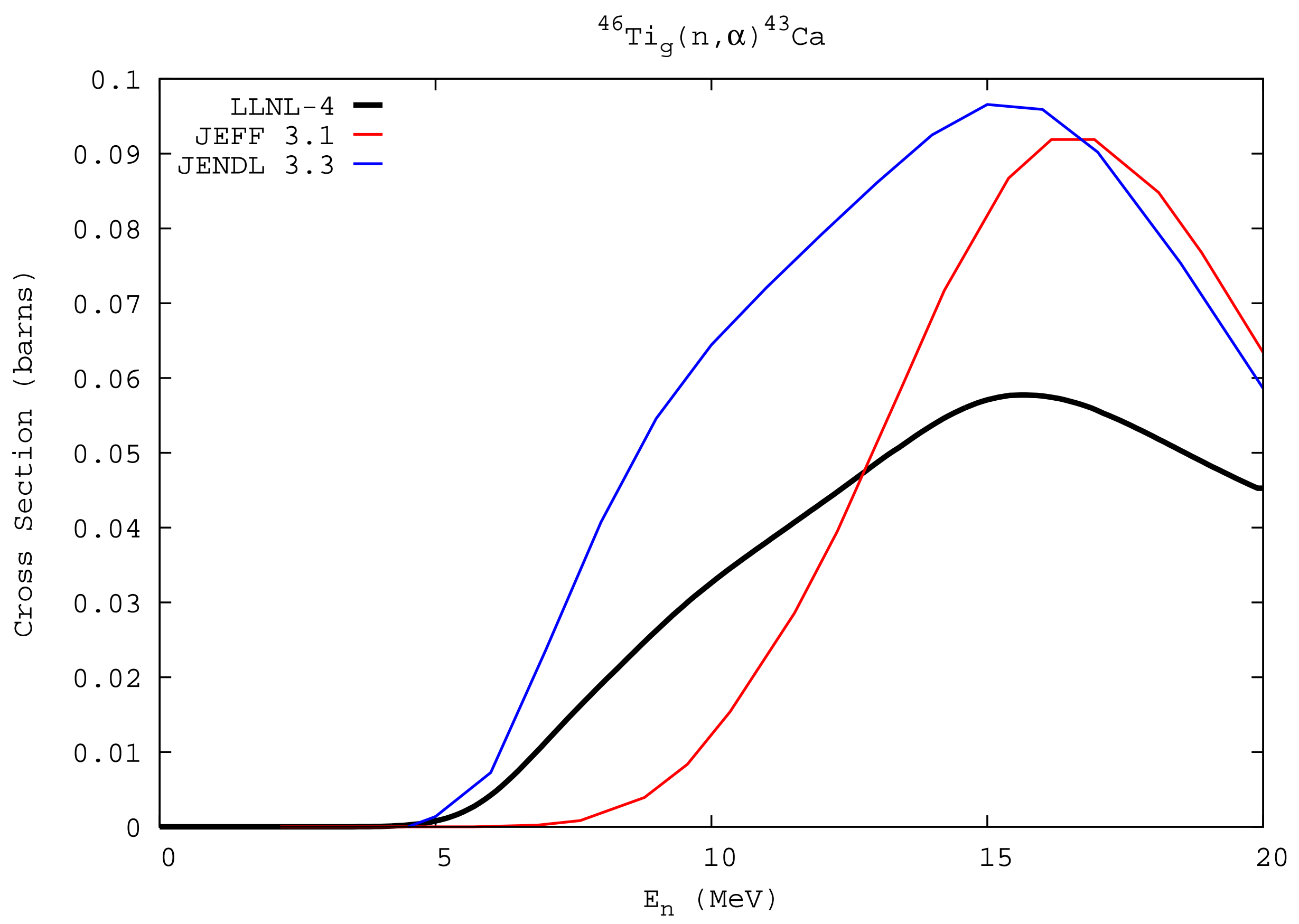


${ }^{46} \mathrm{Ti}_{\mathrm{g}}(\mathrm{n}, \mathrm{d}){ }^{45} \mathrm{SC}$

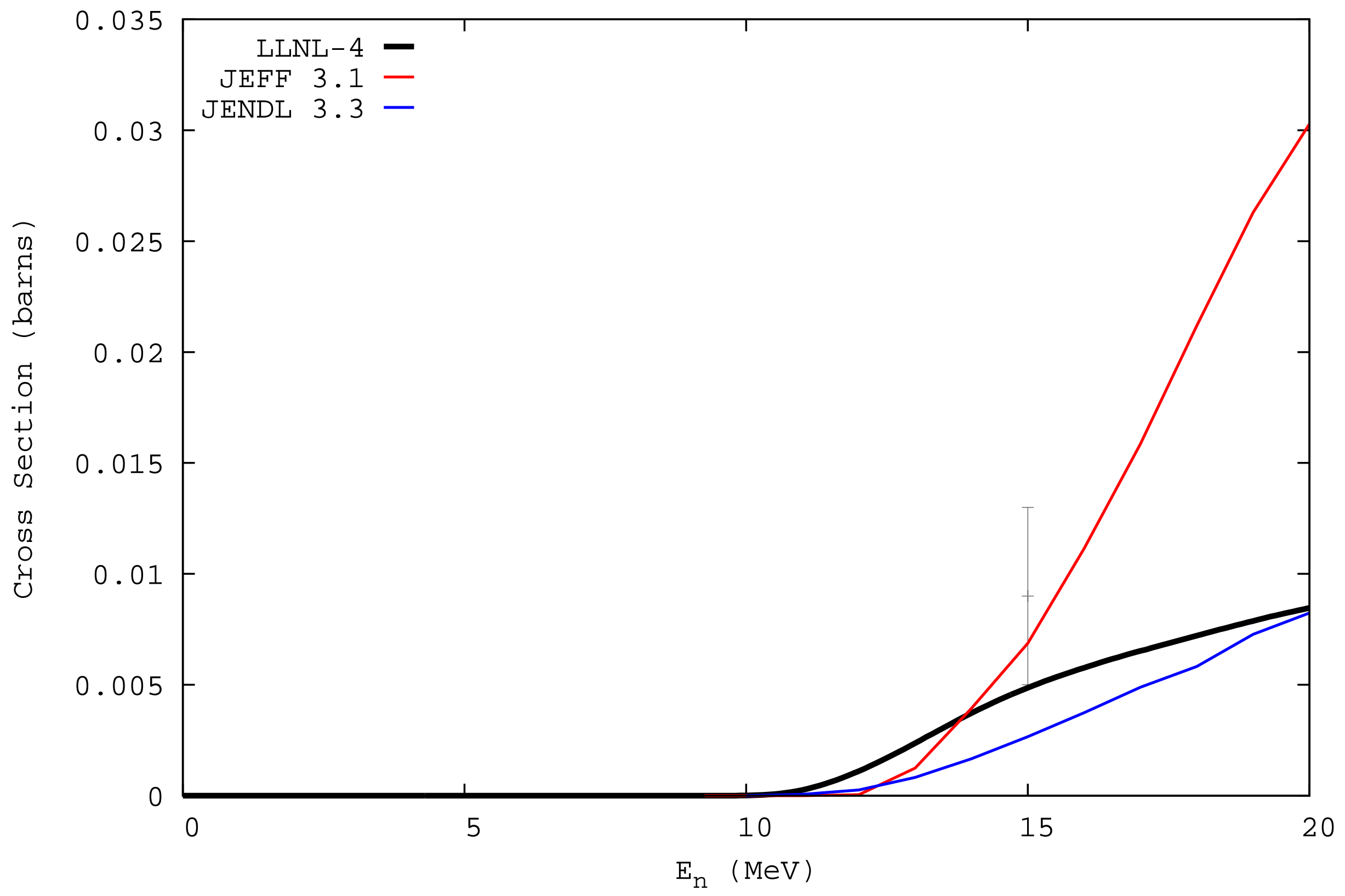




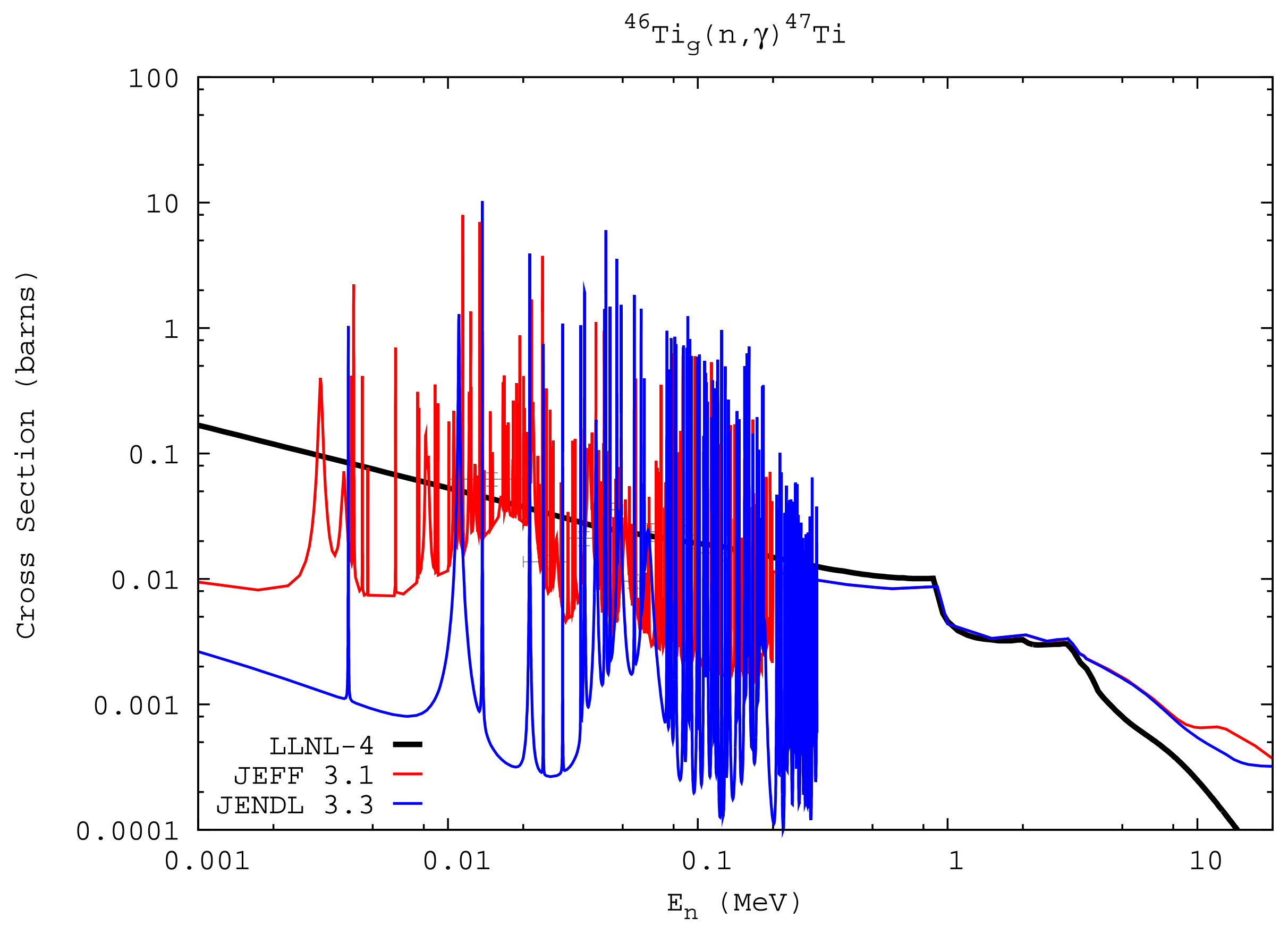




$$
{ }^{46} \mathrm{Ti}_{\mathrm{g}}(\mathrm{n}, \mathrm{n}){ }^{46} \mathrm{Ti}
$$

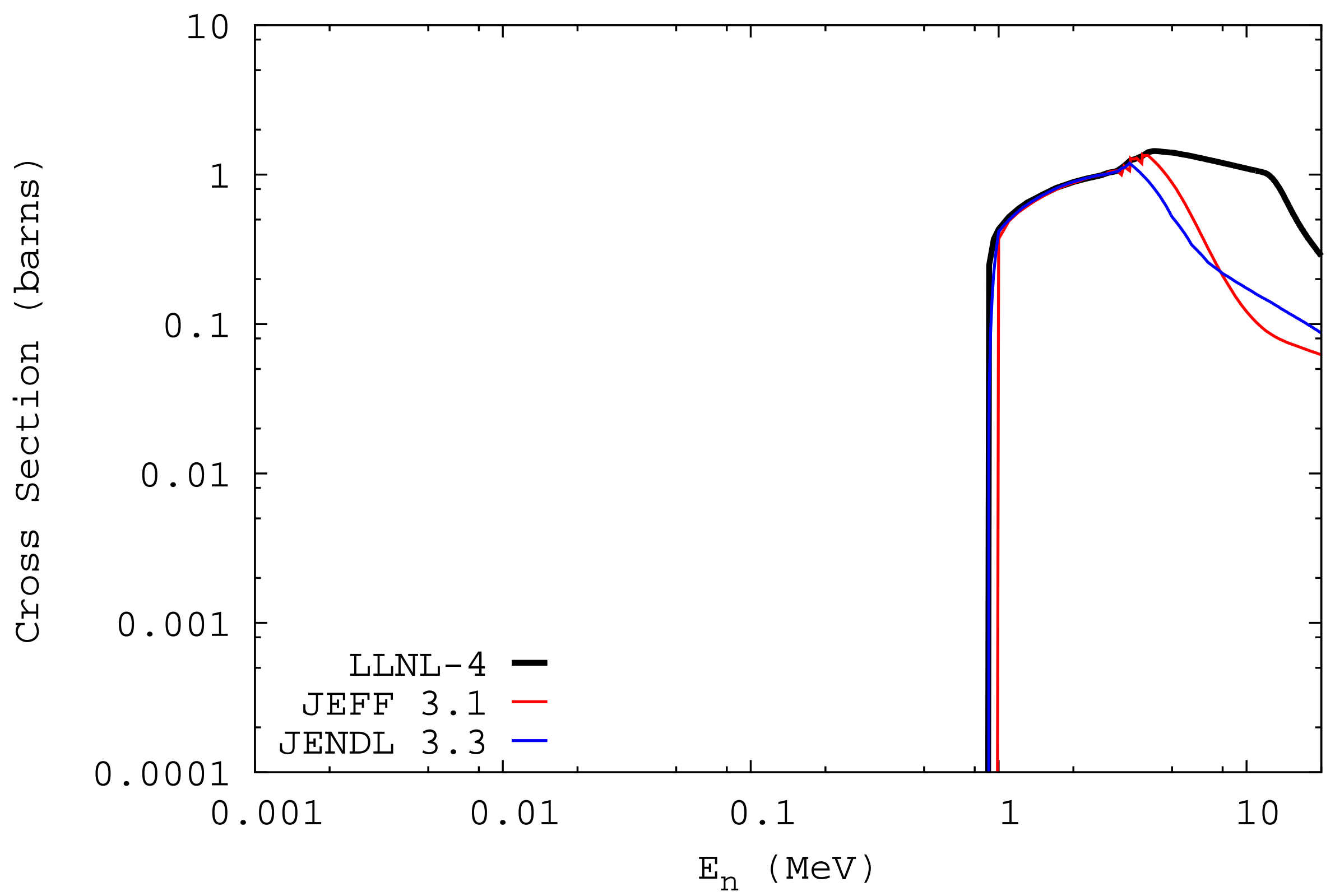


${ }^{46} \mathrm{Ti}_{g}(\mathrm{n}, \mathrm{np})+(\mathrm{n}, \mathrm{pn}){ }^{45} \mathrm{SC}$

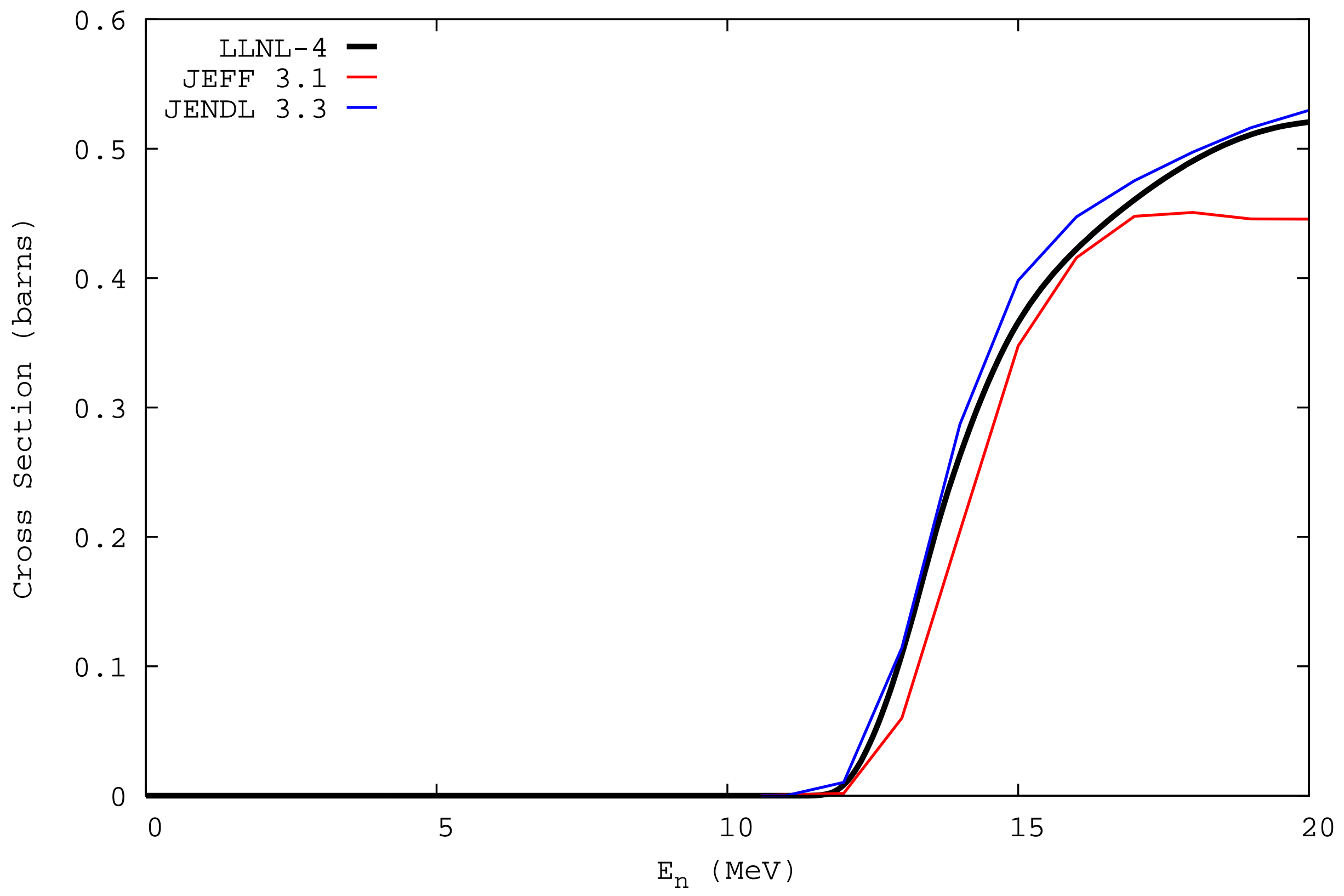




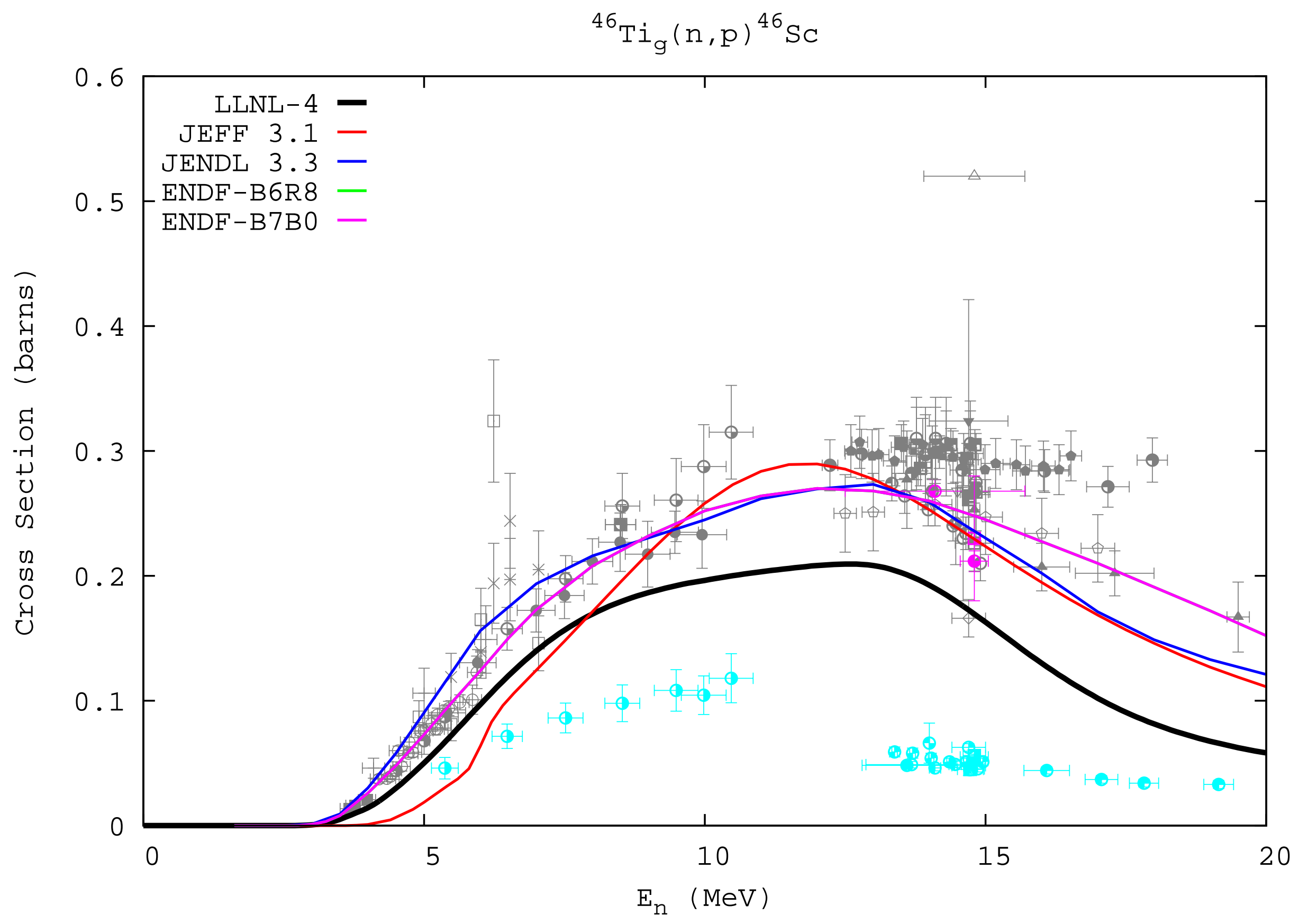


${ }^{47} \mathrm{Ti}_{g}(\mathrm{n}, 2 \mathrm{n}){ }^{46} \mathrm{Ti}$

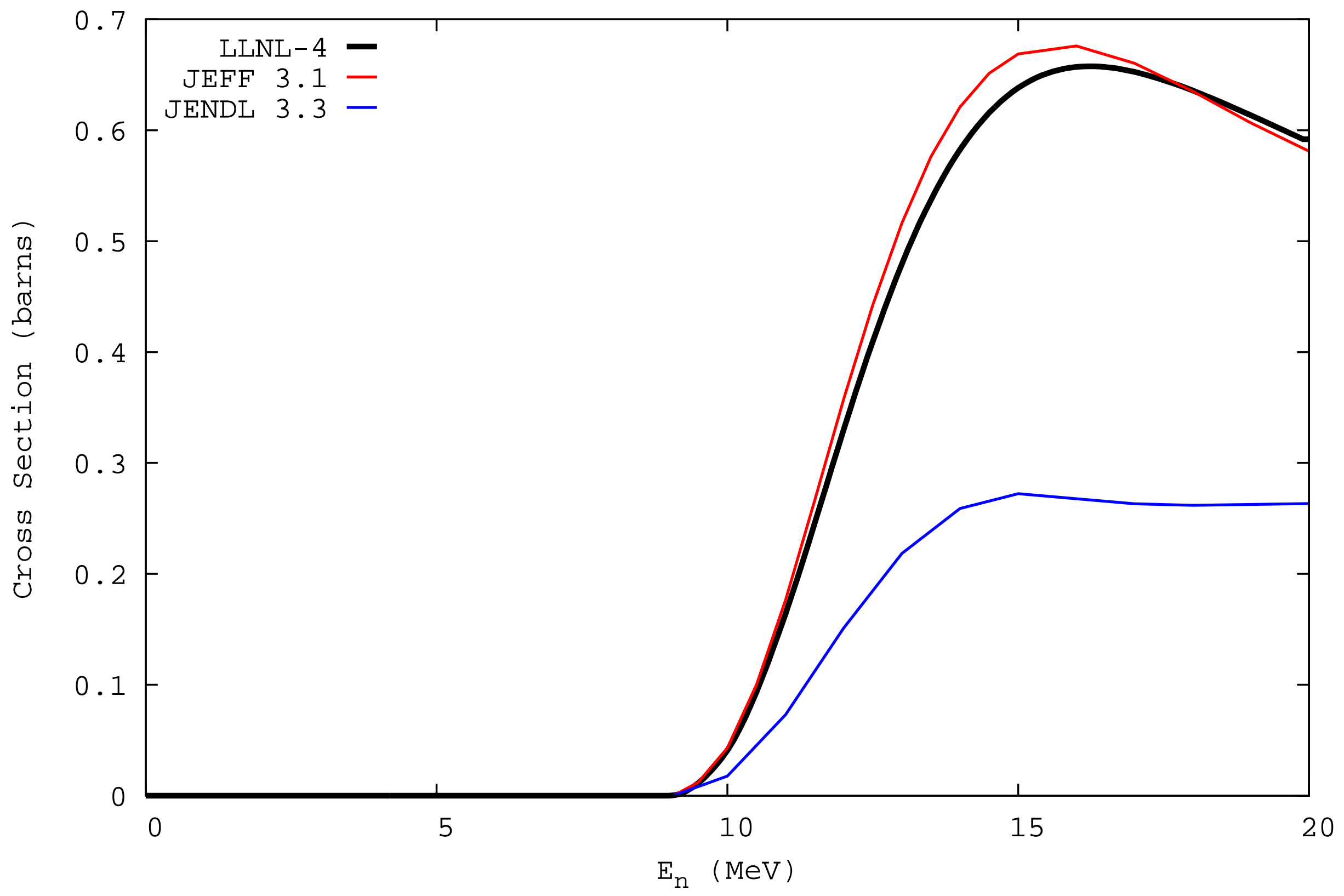




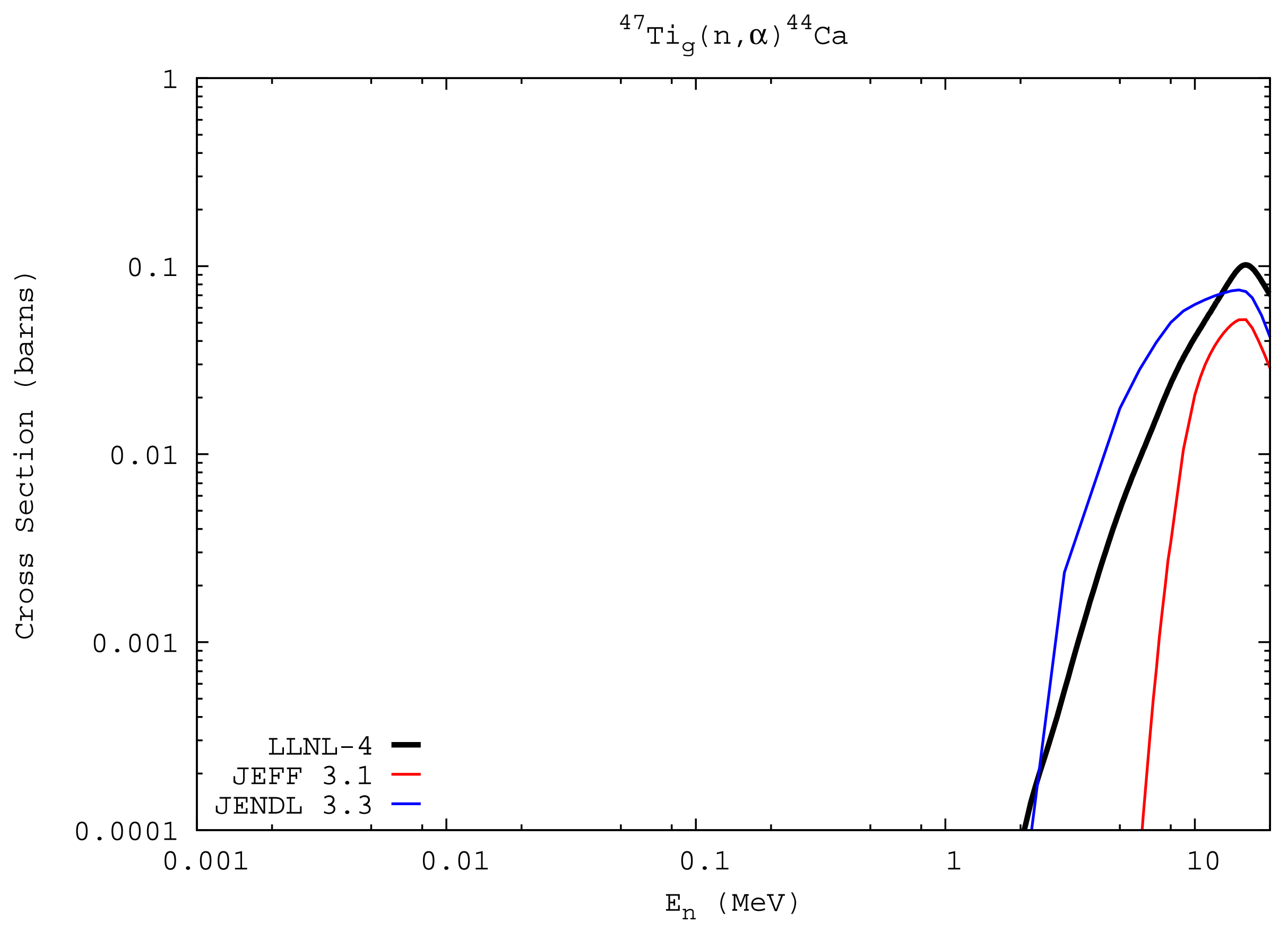


${ }^{47} \mathrm{Ti}_{\mathrm{g}}(\mathrm{n}, \mathrm{d}){ }^{46} \mathrm{SC}$

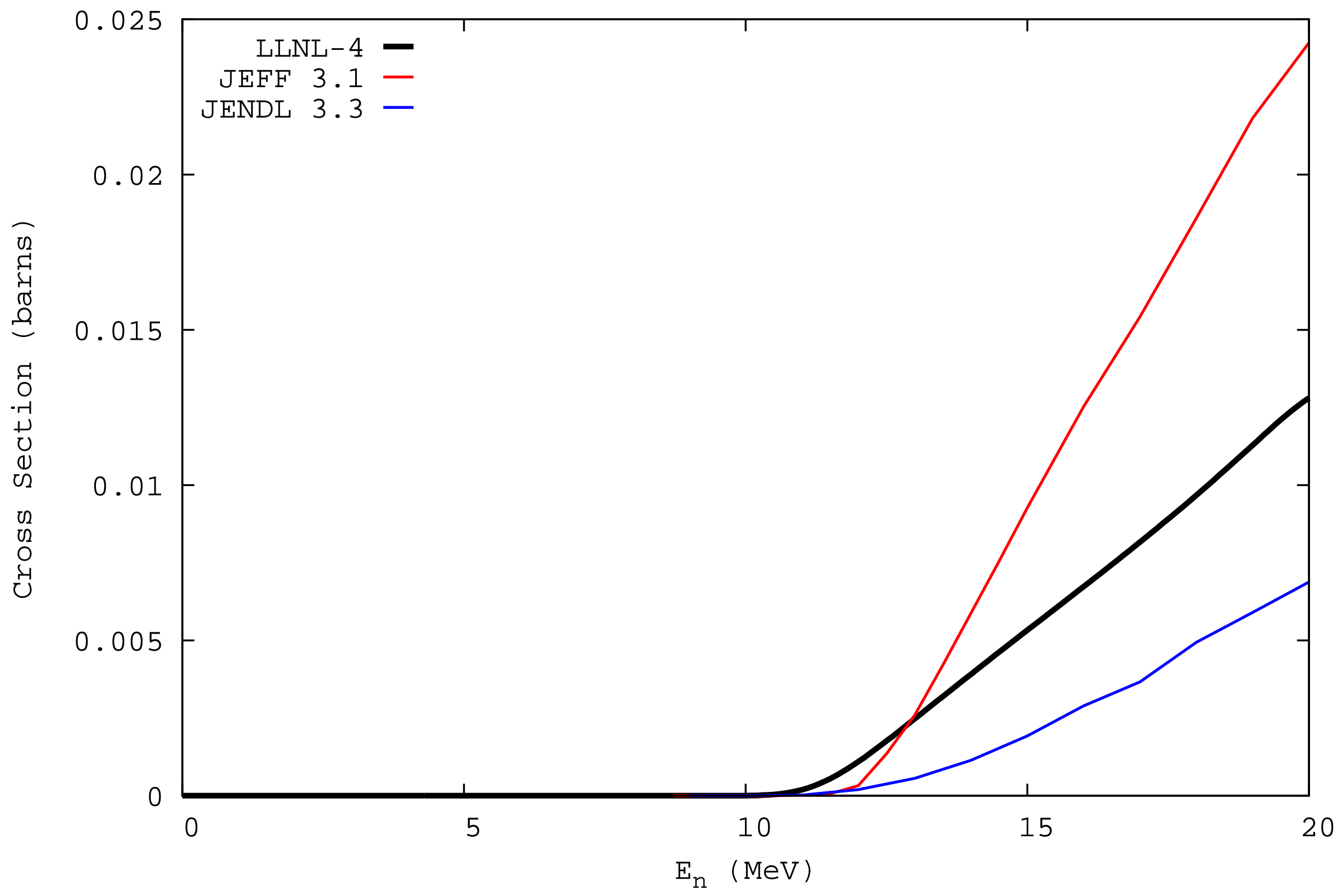




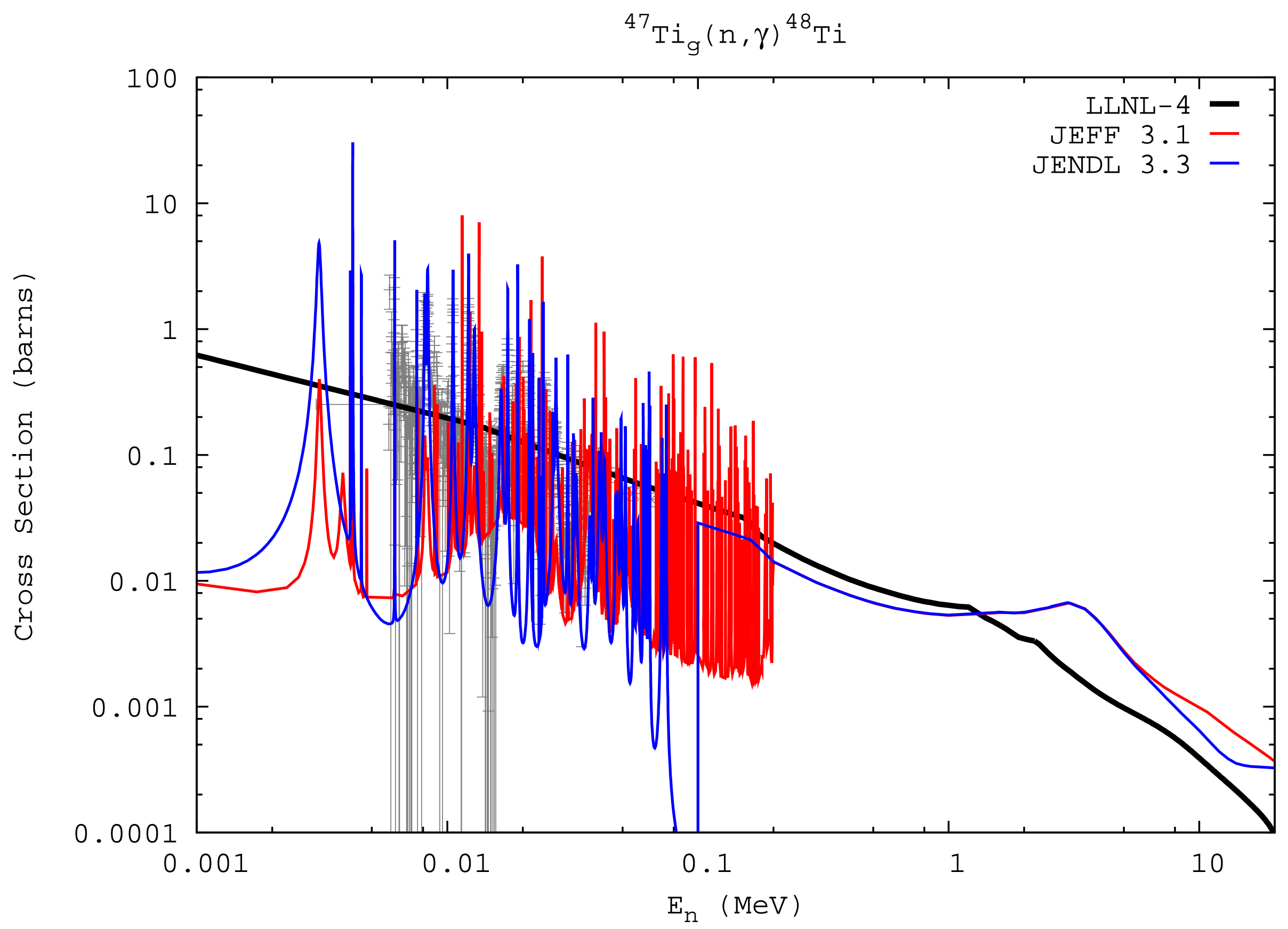




$$
{ }^{47} \mathrm{Ti}_{\mathrm{g}}(\mathrm{n}, \mathrm{n}){ }^{47} \mathrm{Ti}
$$

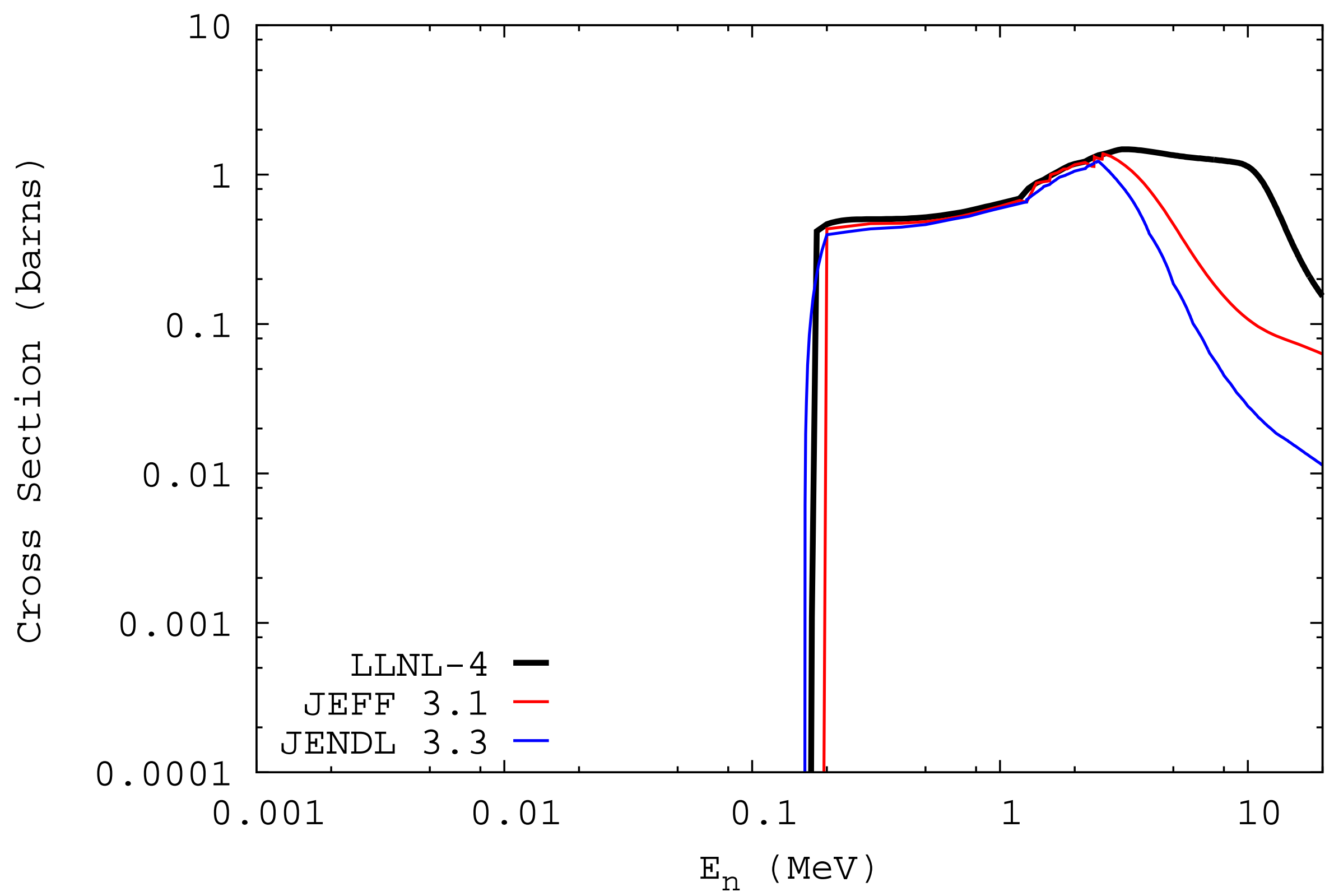


${ }^{47} \mathrm{Ti}_{g}(\mathrm{n}, \mathrm{np})+(\mathrm{n}, \mathrm{pn}){ }^{46} \mathrm{Sc}$

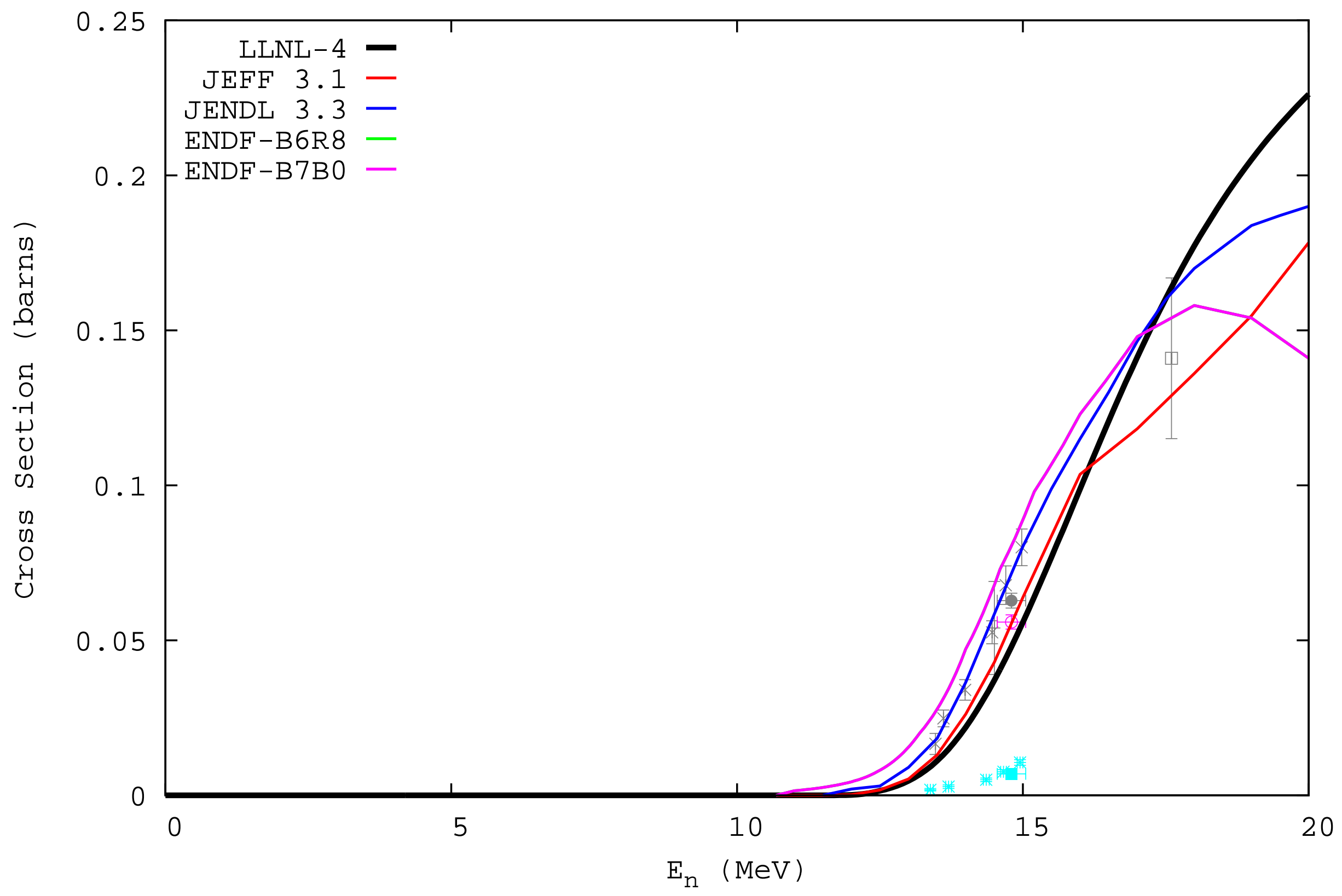




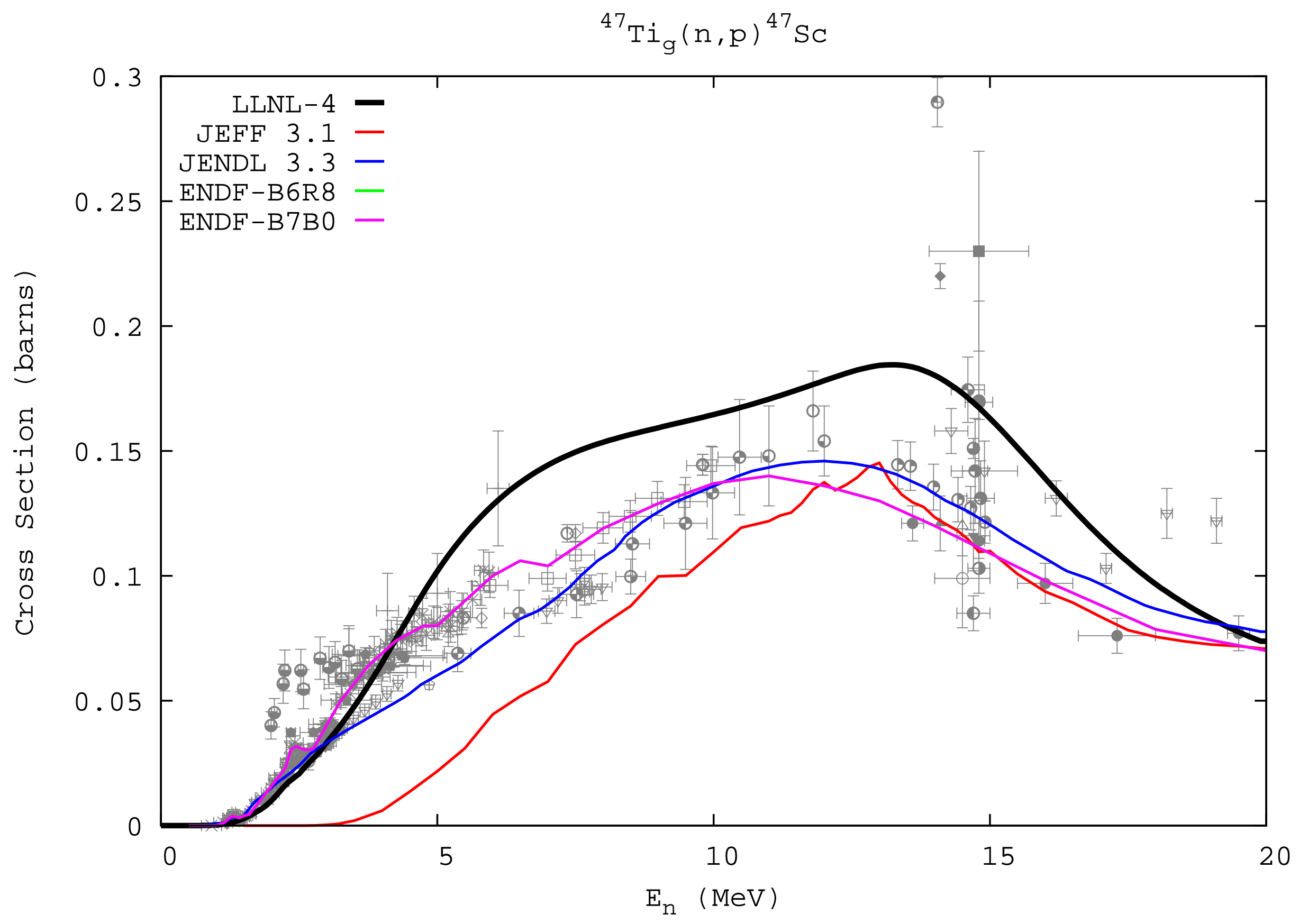


${ }^{48} \mathrm{Ti}_{g}(\mathrm{n}, 2 \mathrm{n}){ }^{47} \mathrm{Ti}$

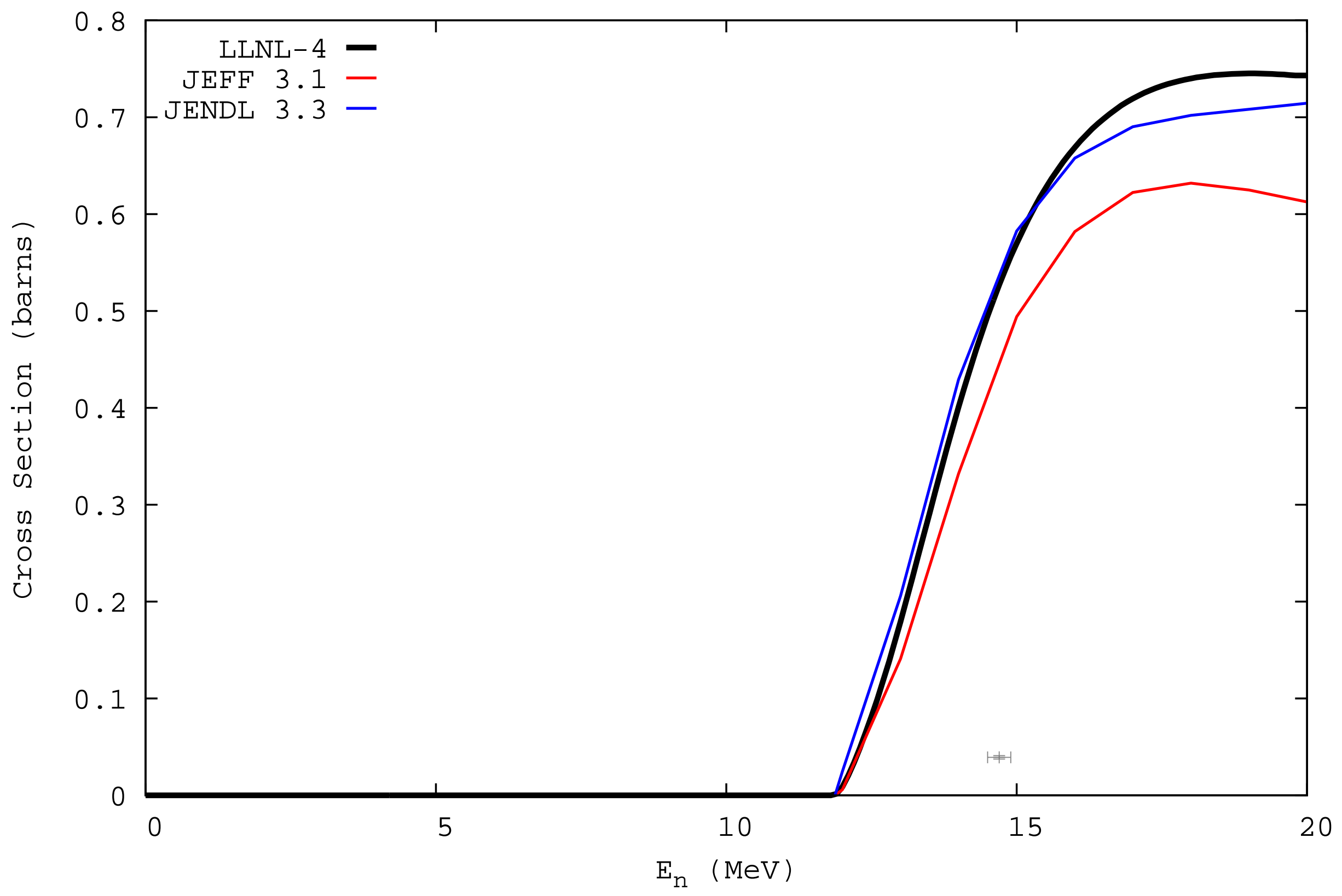


${ }^{48} \mathrm{Ti}_{\mathrm{g}}(\mathrm{n}, \alpha){ }^{45} \mathrm{Ca}$

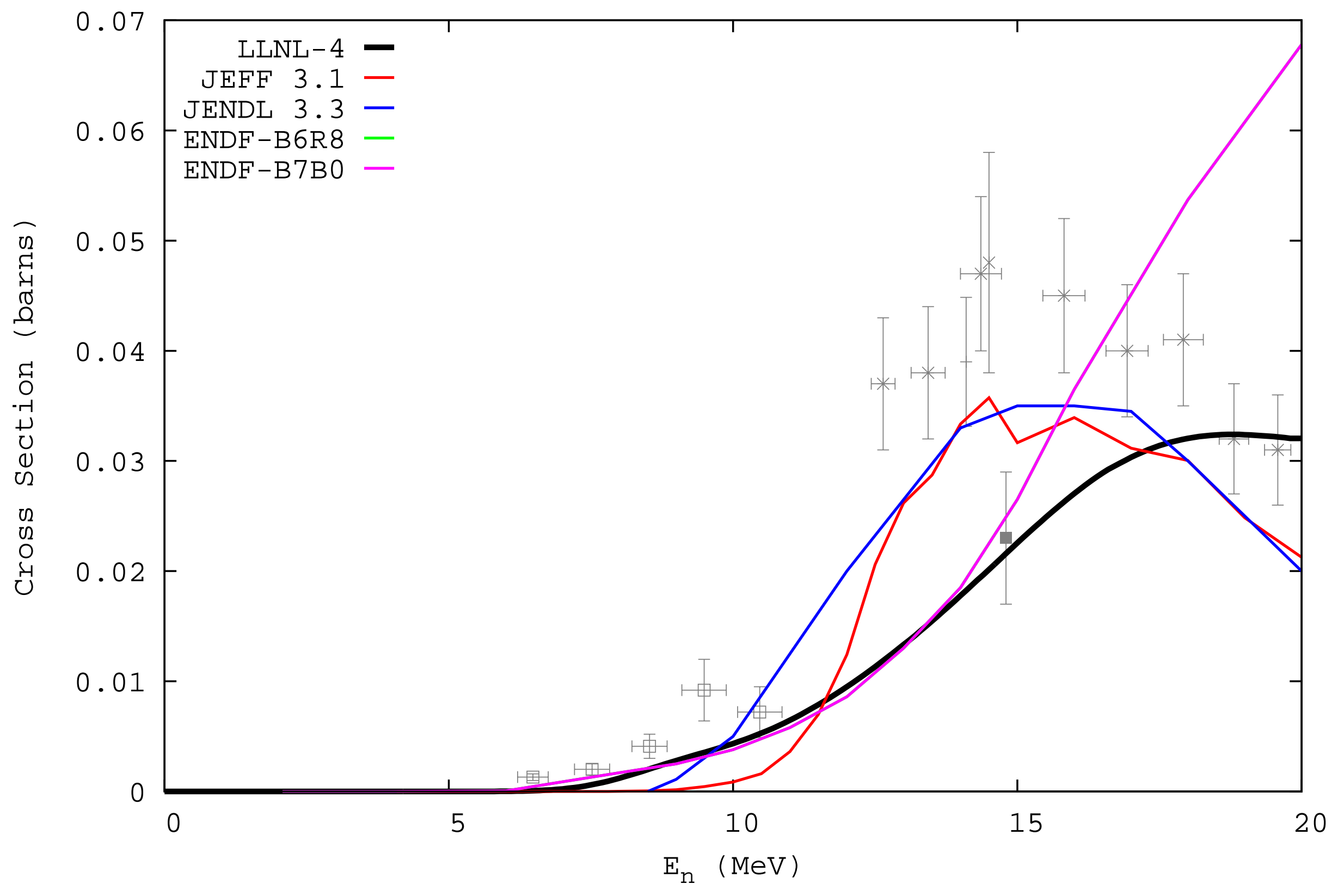


${ }^{48} \mathrm{Ti}_{\mathrm{g}}(\mathrm{n}, \mathrm{d}){ }^{47} \mathrm{SC}$

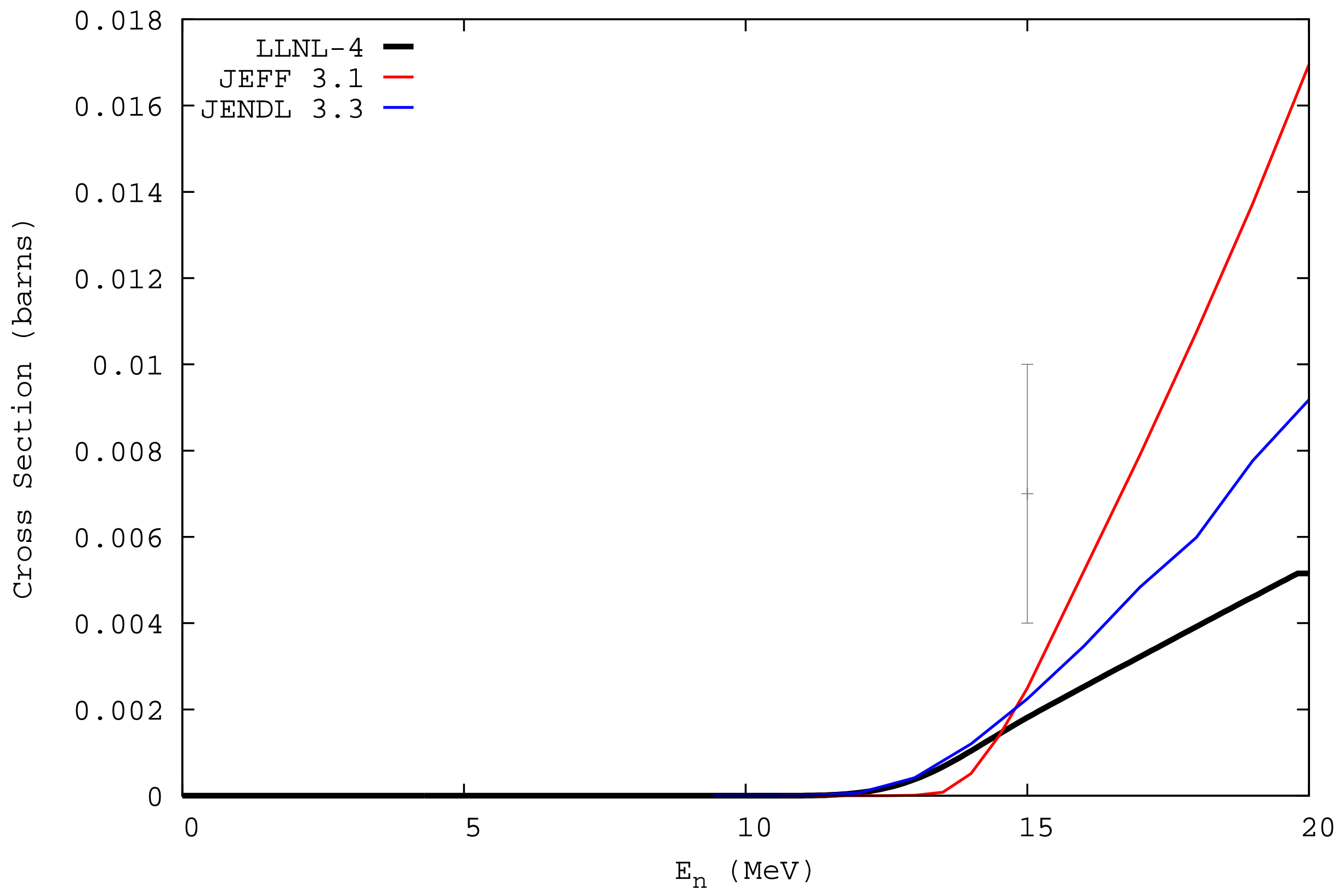




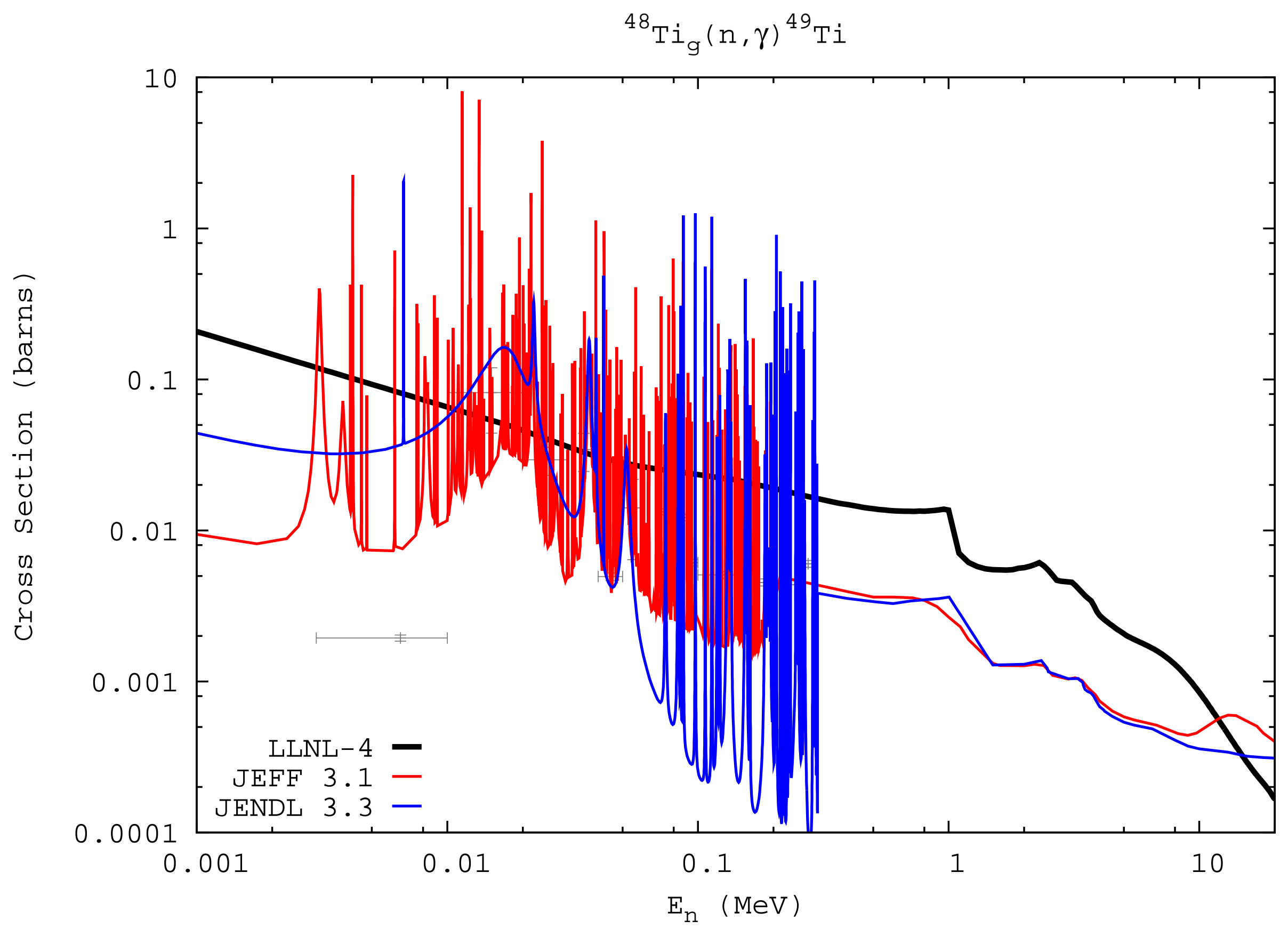




$$
{ }^{48} \mathrm{Ti}_{\mathrm{g}}(\mathrm{n}, \mathrm{n}){ }^{48} \mathrm{Ti}
$$

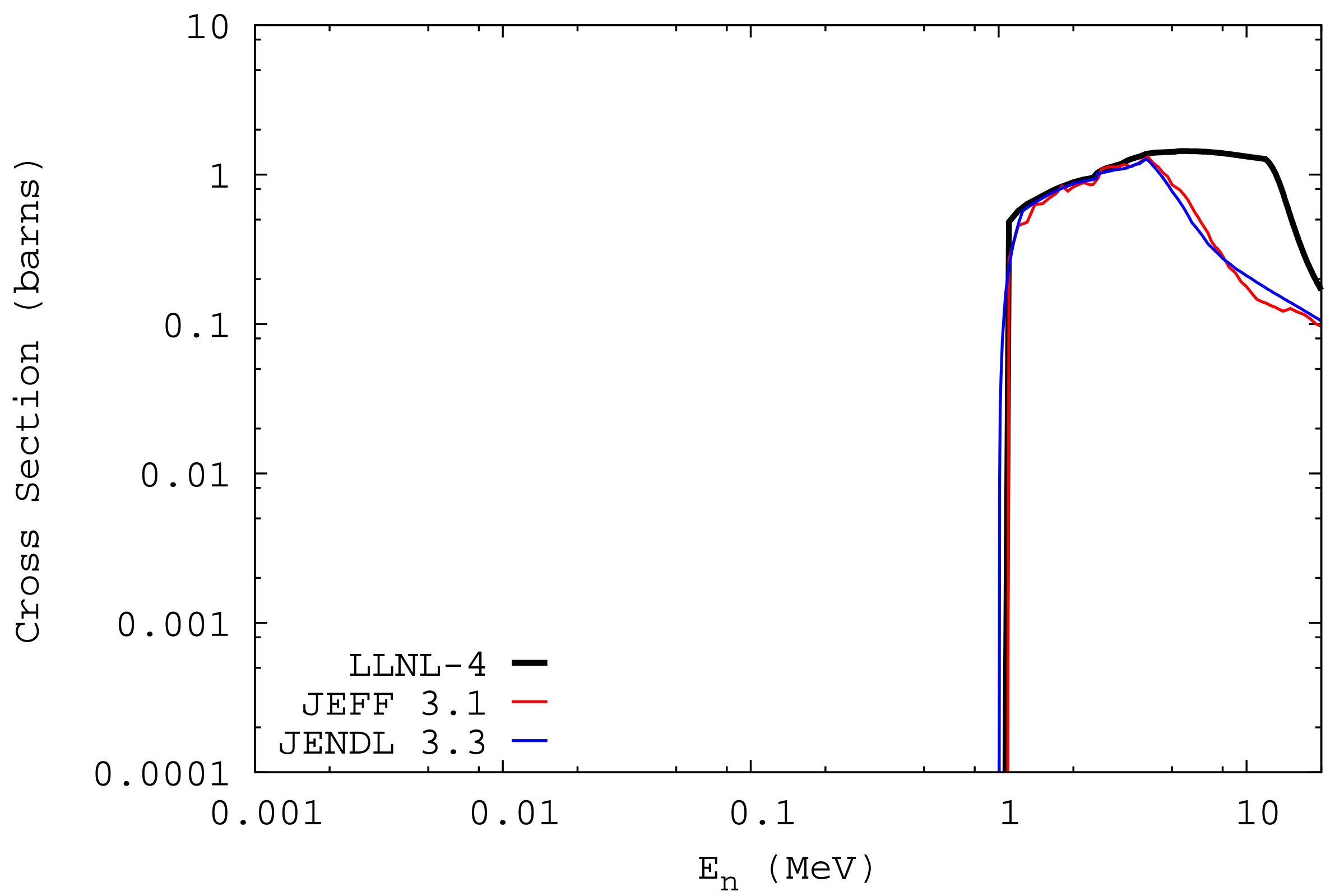


${ }^{48} \mathrm{Ti}_{g}(\mathrm{n}, \mathrm{np})+(\mathrm{n}, \mathrm{pn})^{47} \mathrm{Sc}$

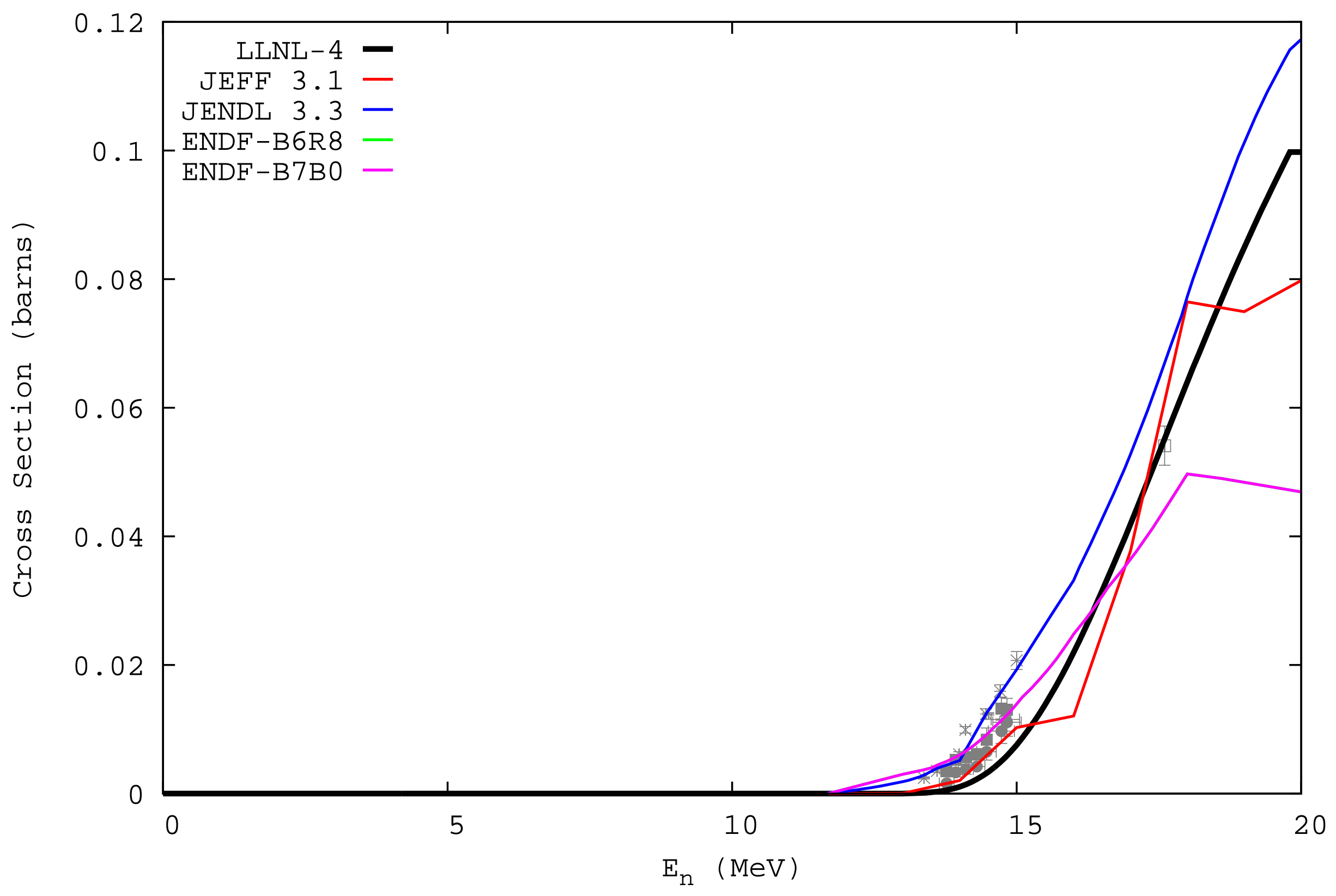


${ }^{48} \mathrm{Ti}_{\mathrm{g}}(\mathrm{n}, \mathrm{p}){ }^{48} \mathrm{SC}$

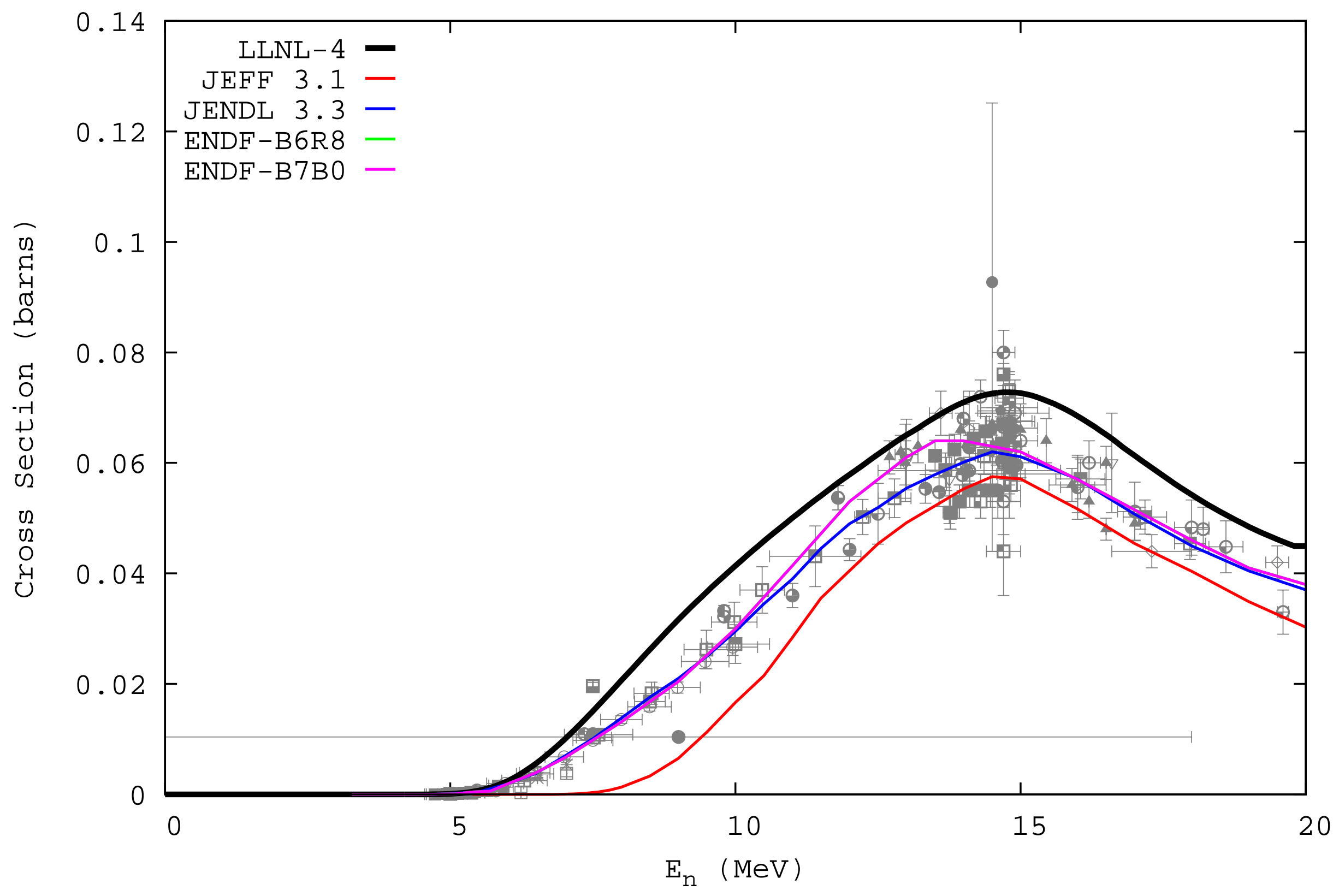




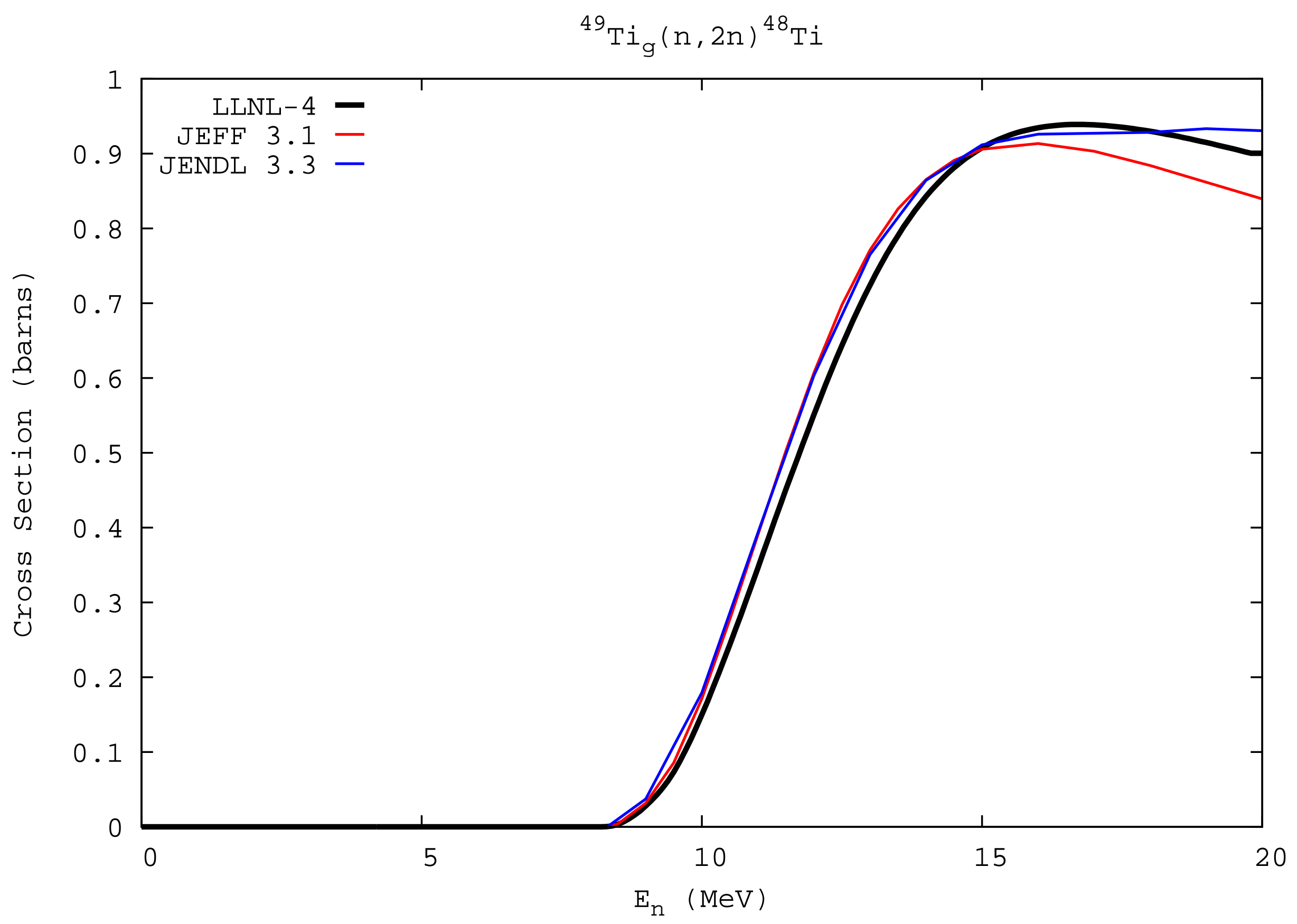




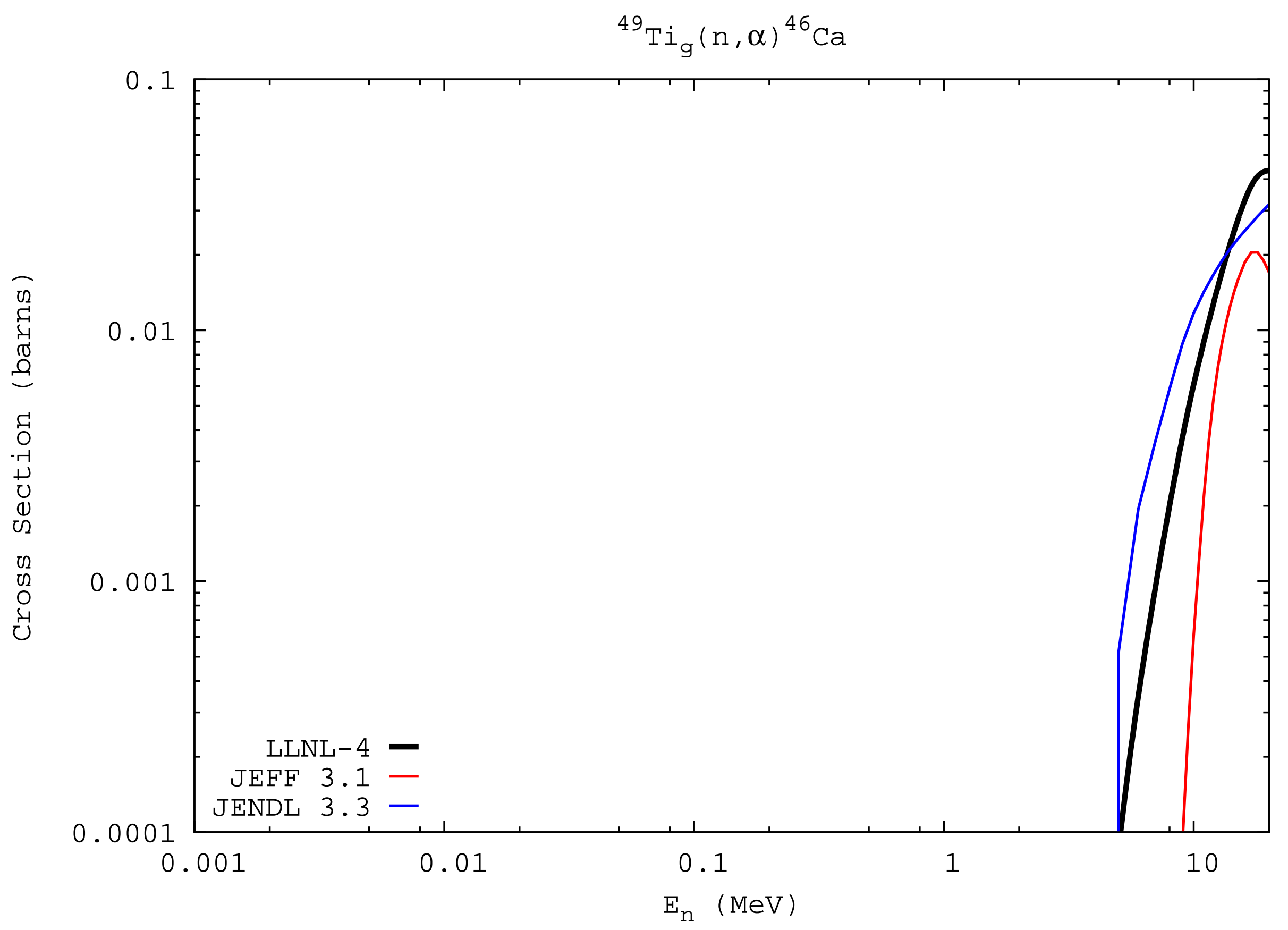


${ }^{49} \mathrm{Ti}_{\mathrm{g}}(\mathrm{n}, \mathrm{d}){ }^{48} \mathrm{SC}$

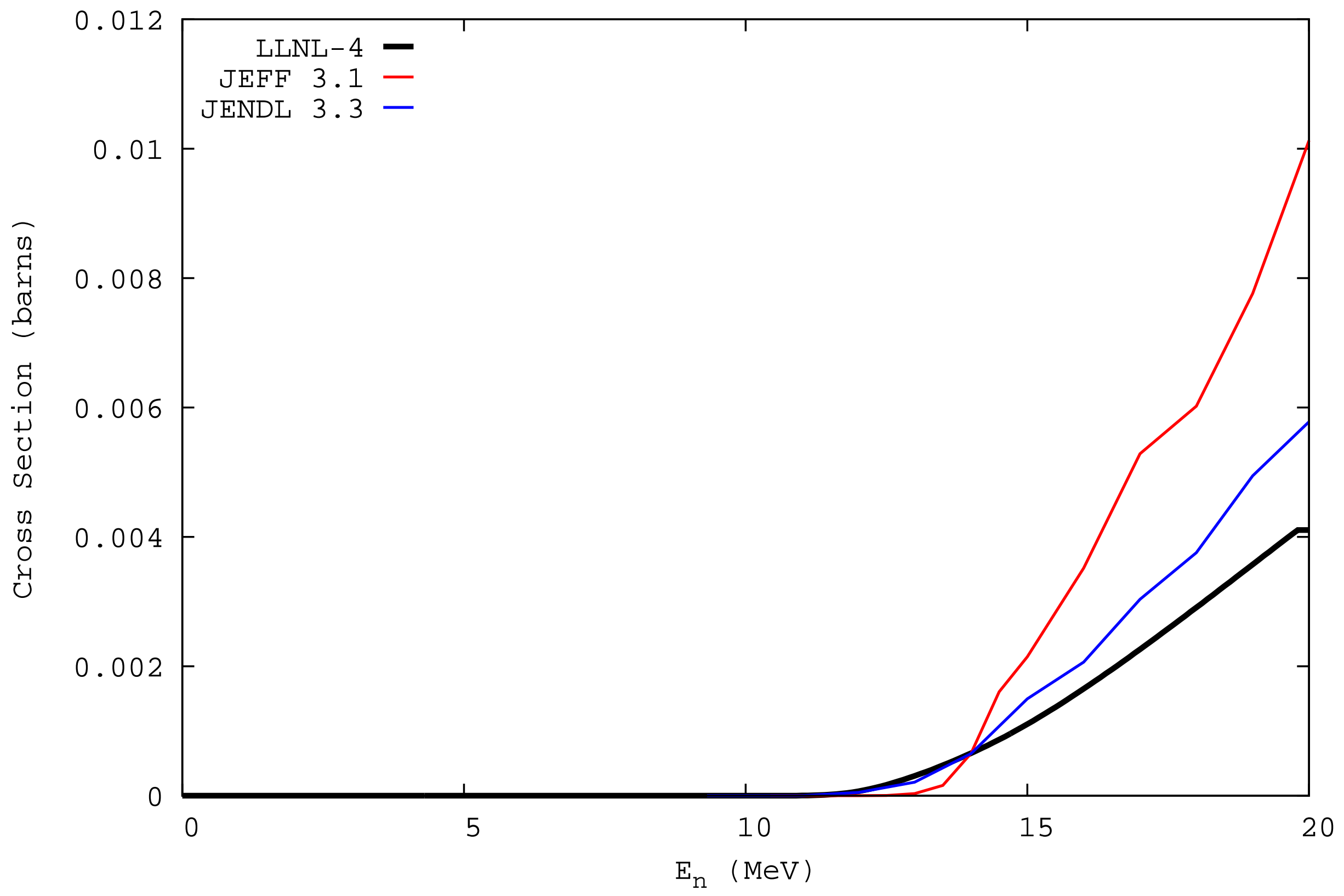




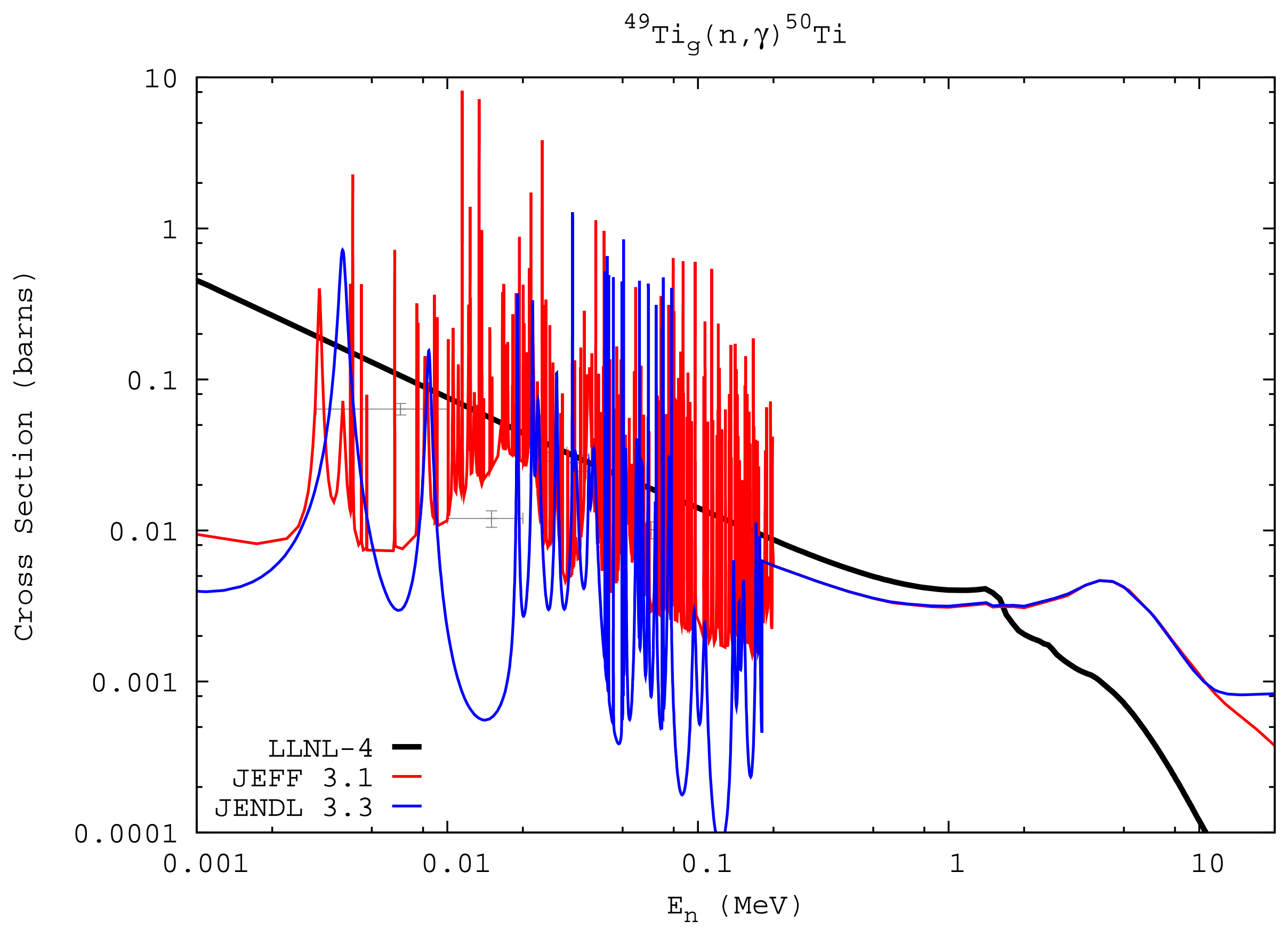




$$
{ }^{49} \mathrm{Ti}_{\mathrm{g}}(\mathrm{n}, \mathrm{n}){ }^{49} \mathrm{Ti}
$$

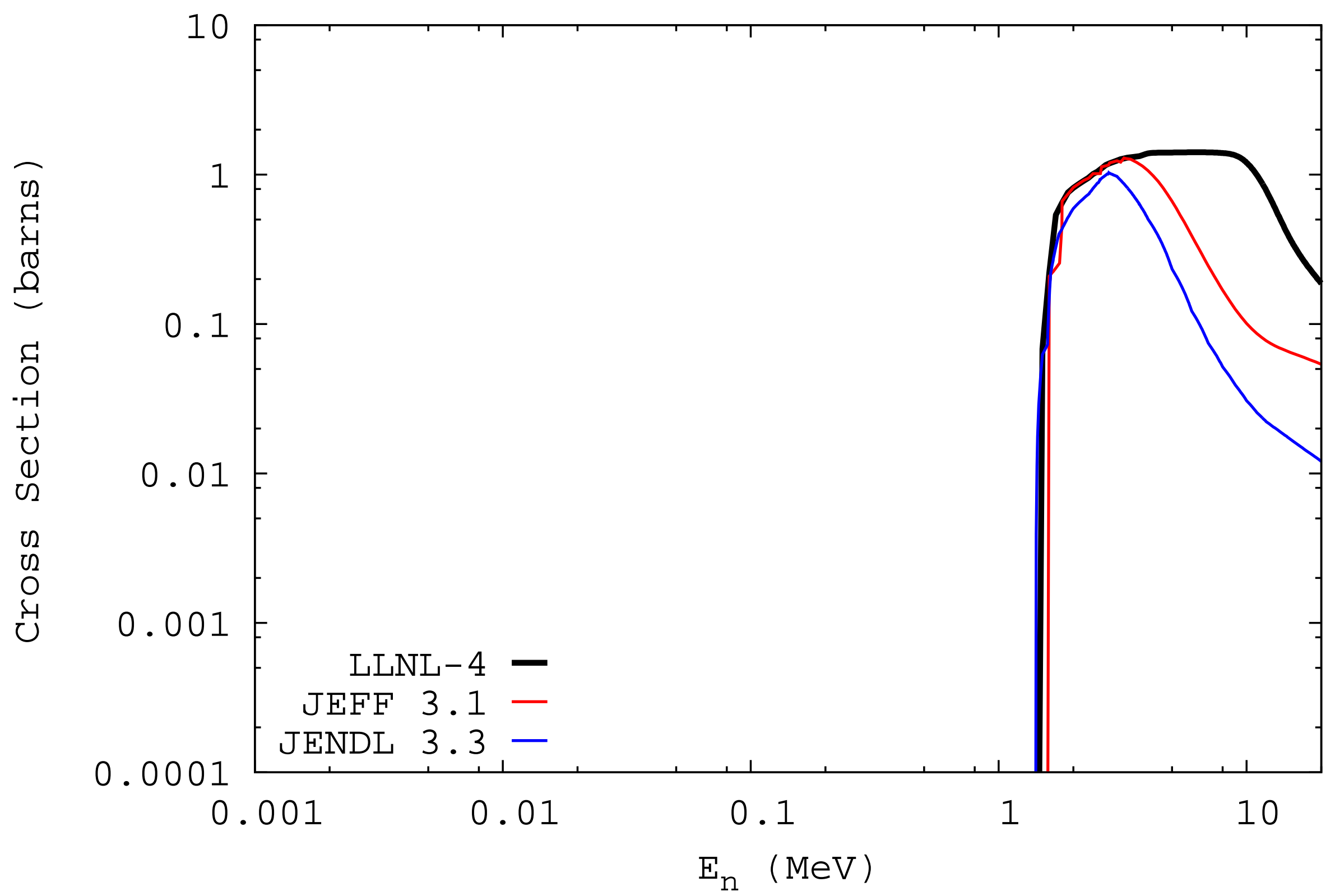


${ }^{49} \mathrm{Ti}_{g}(\mathrm{n}, \mathrm{np})+(\mathrm{n}, \mathrm{pn}){ }^{48} \mathrm{Sc}$

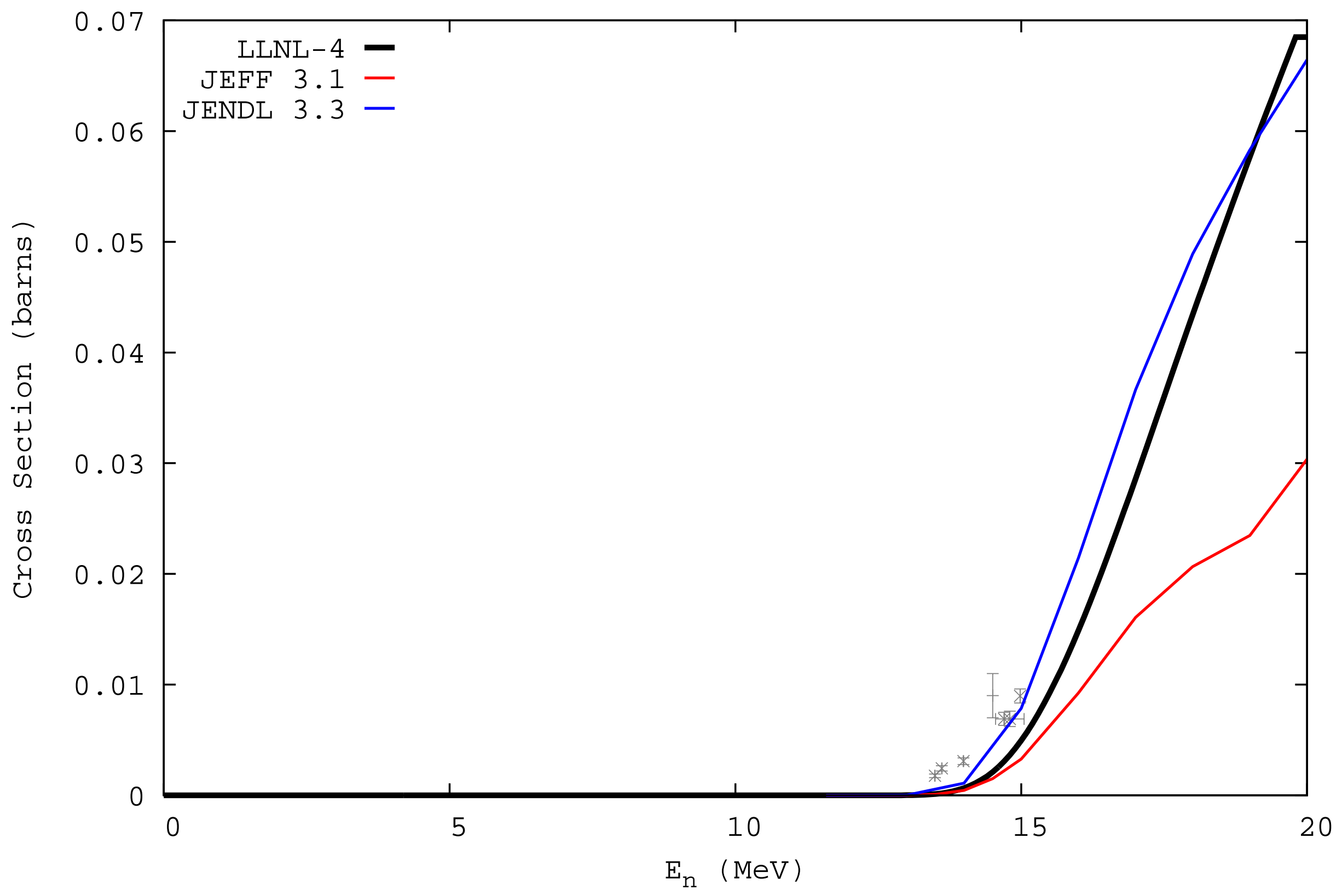




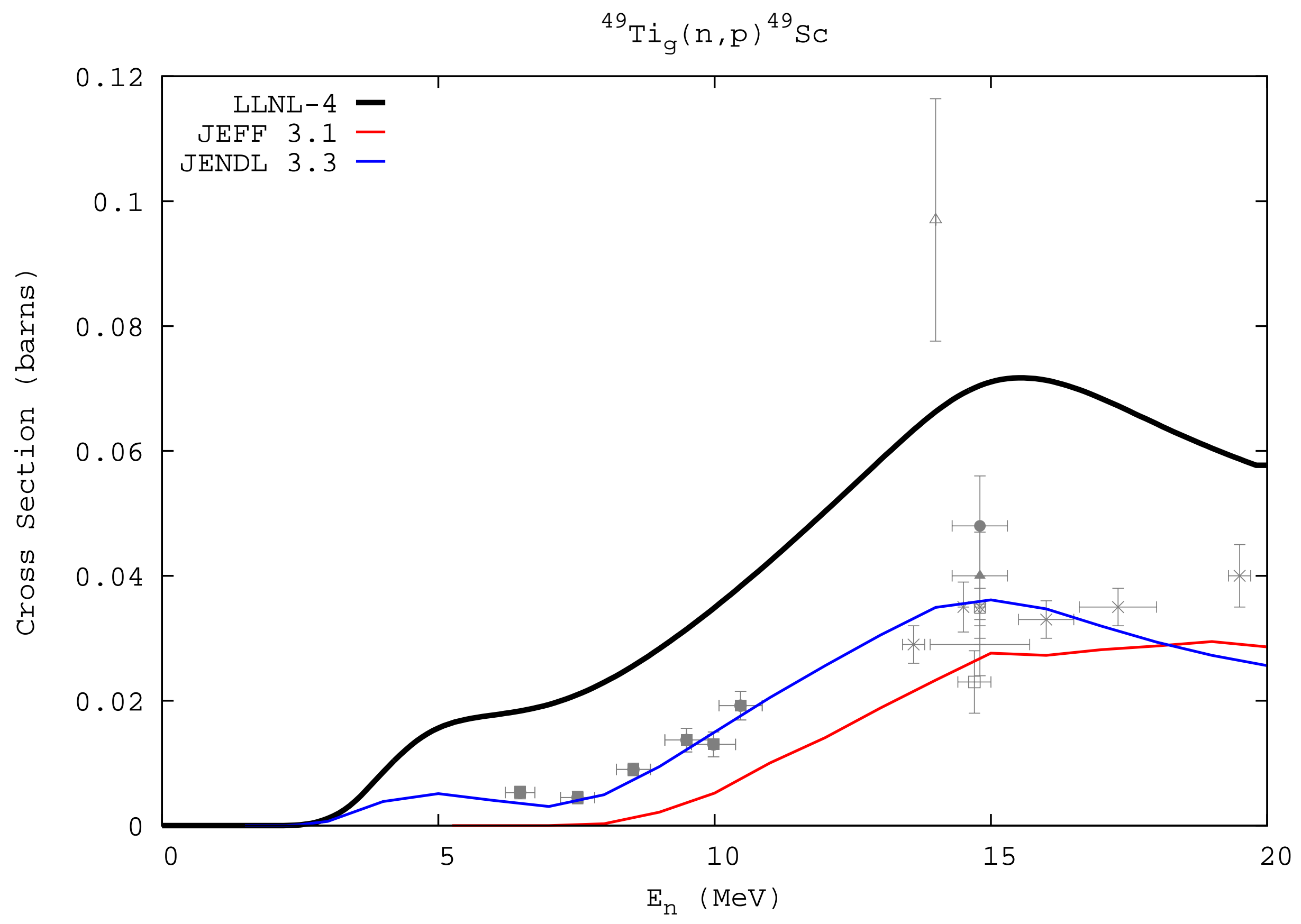


${ }^{50} \mathrm{Ti}_{g}(\mathrm{n}, 2 \mathrm{n}){ }^{49} \mathrm{Ti}$

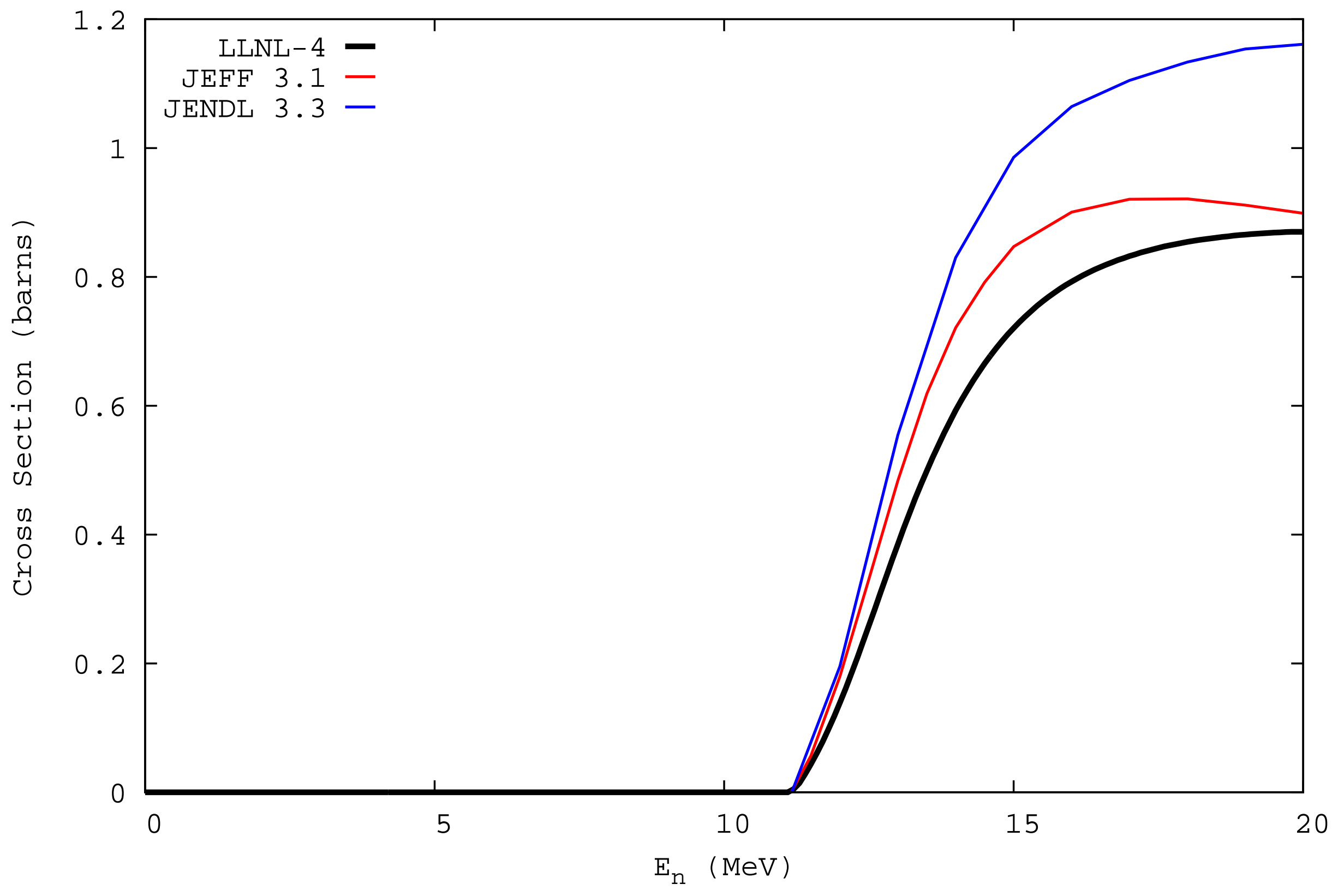




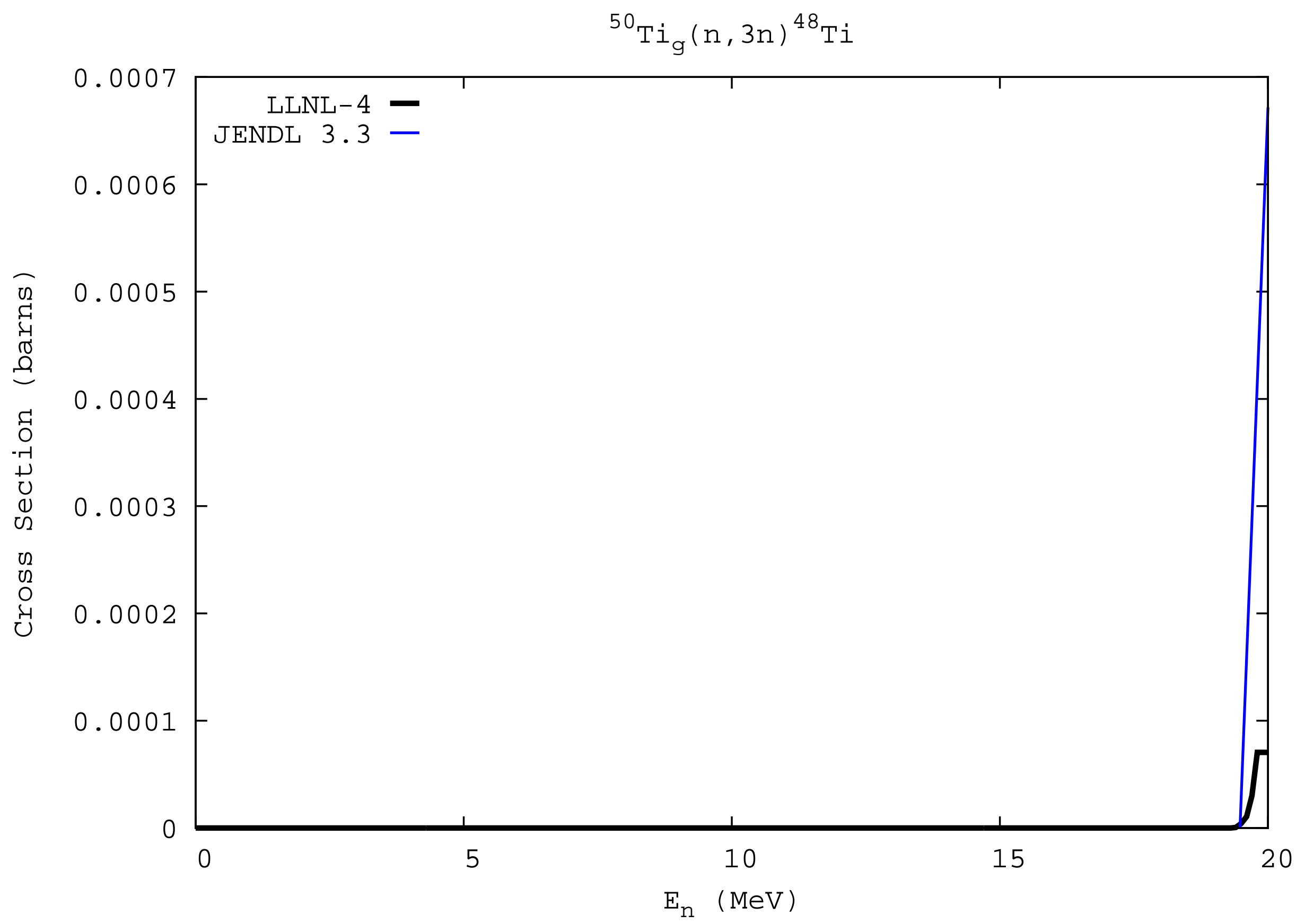




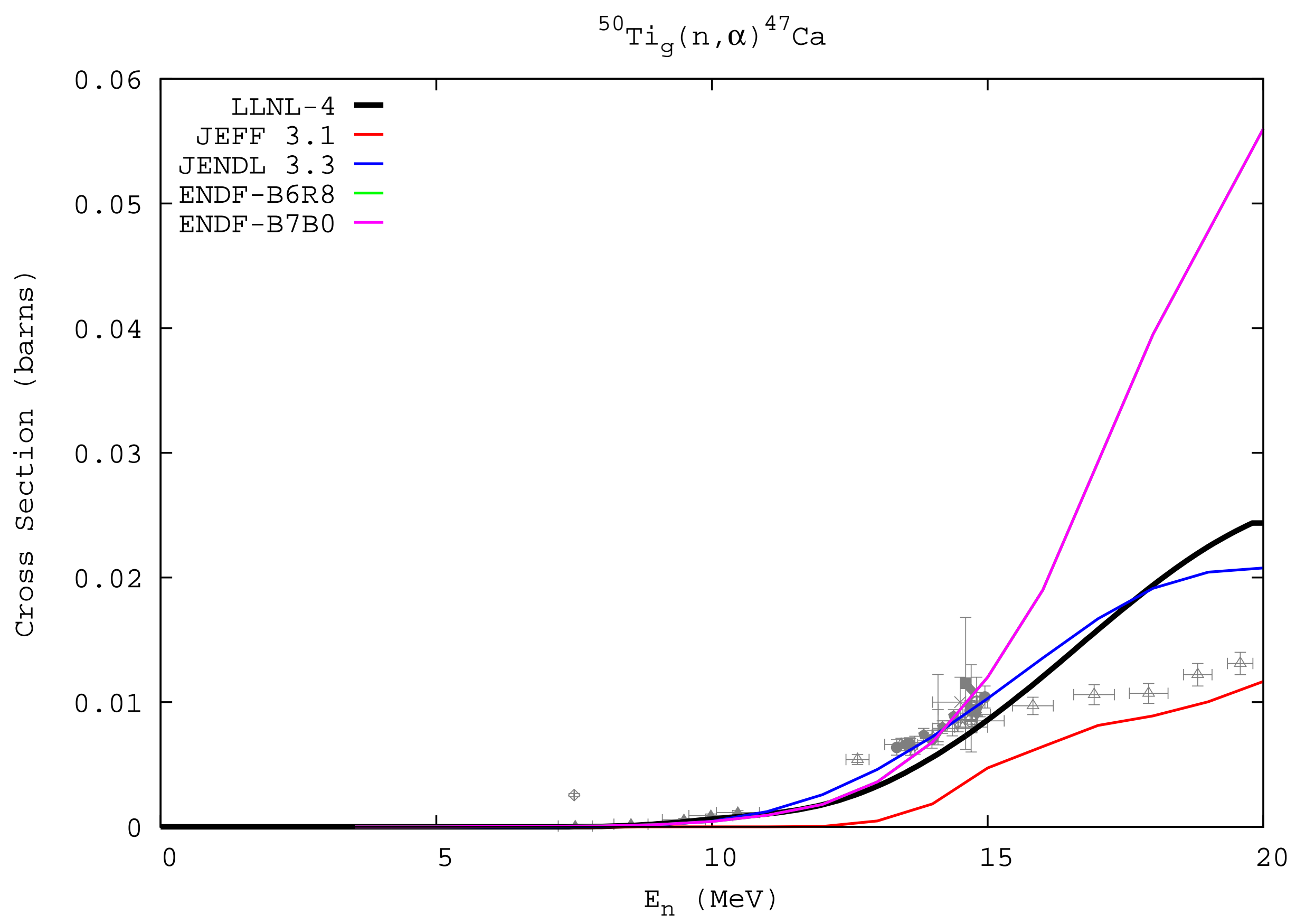




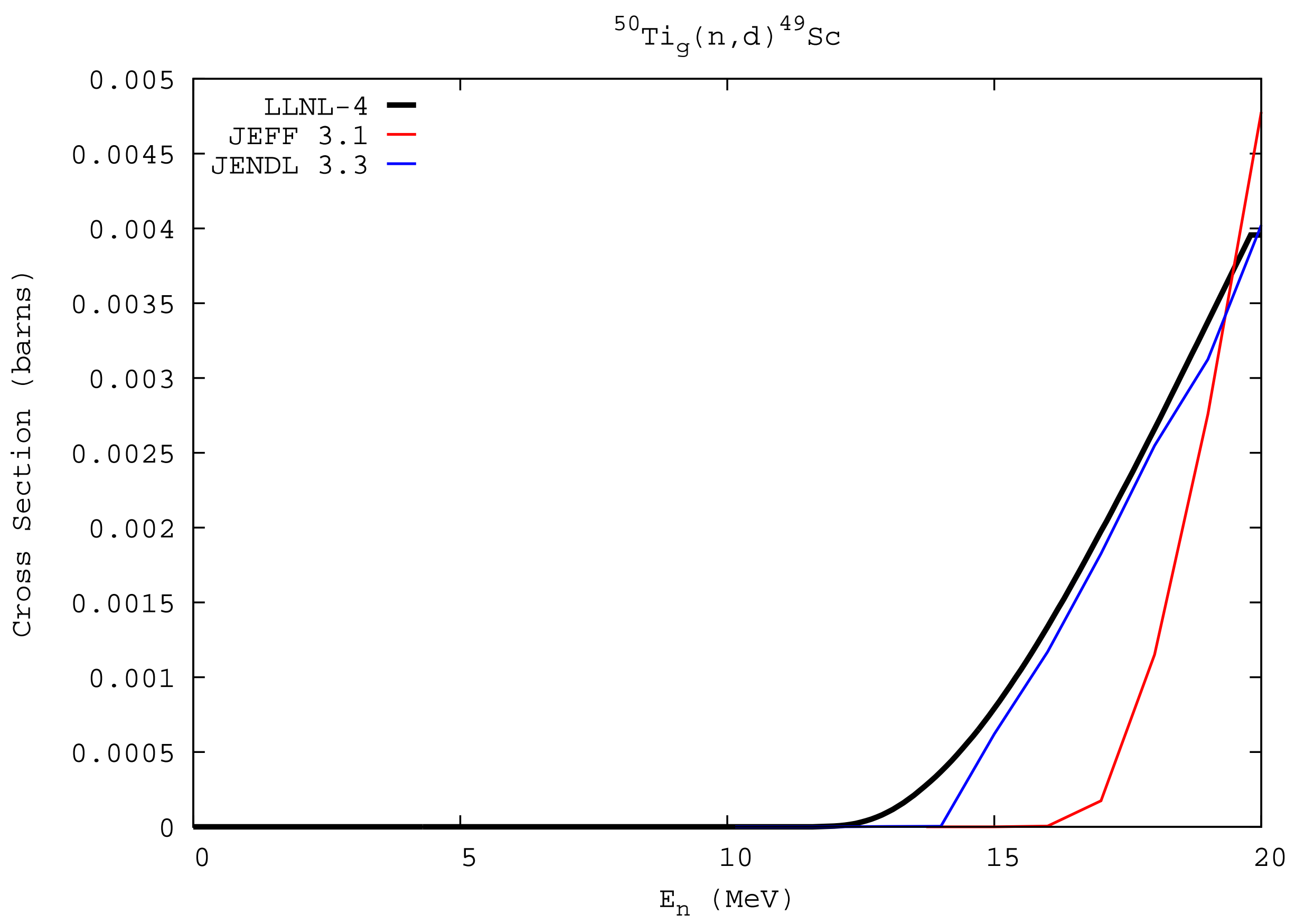




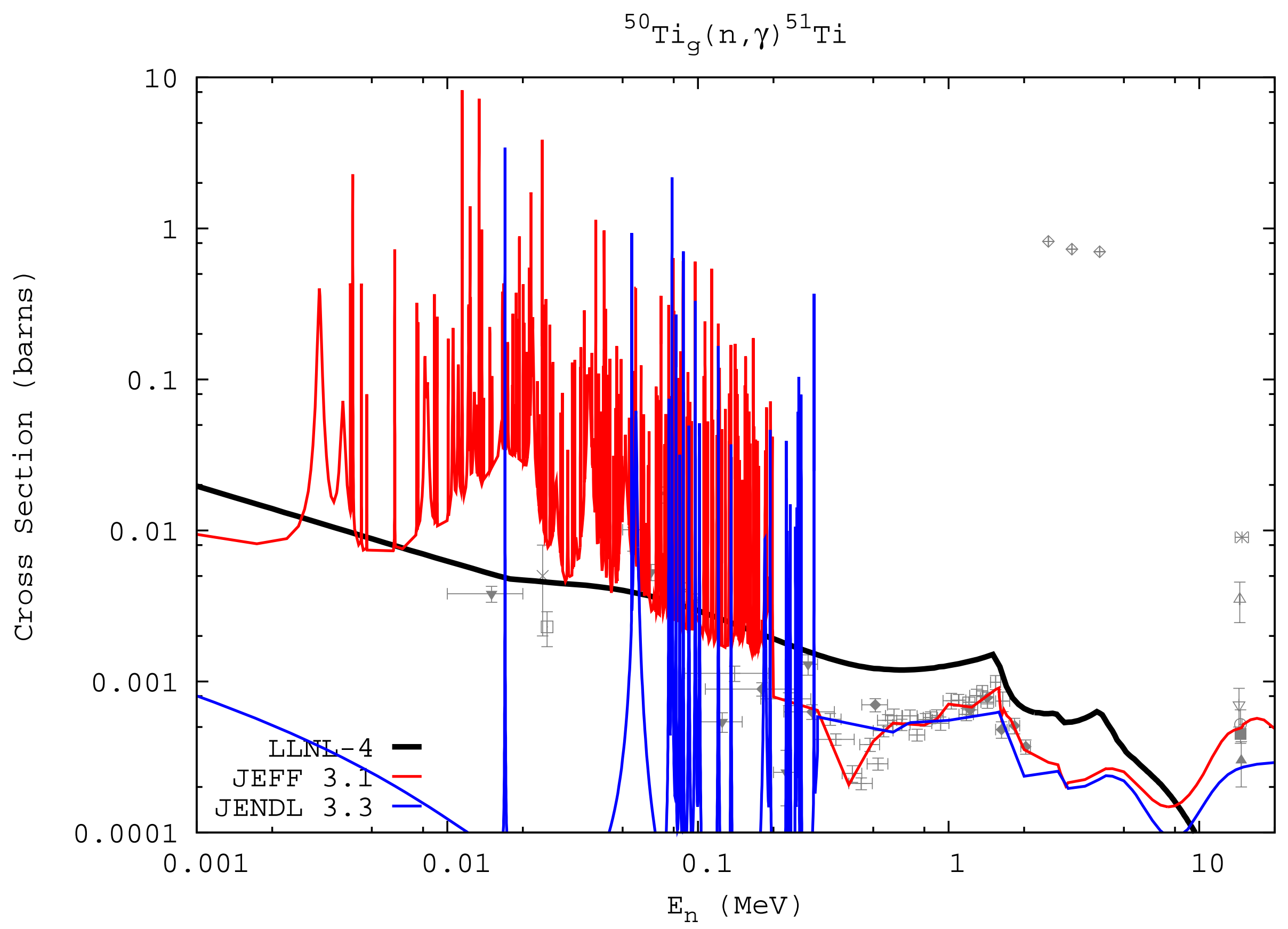




$$
{ }^{50} \mathrm{Ti}_{\mathrm{g}}(\mathrm{n}, \mathrm{n}){ }^{50} \mathrm{Ti}
$$

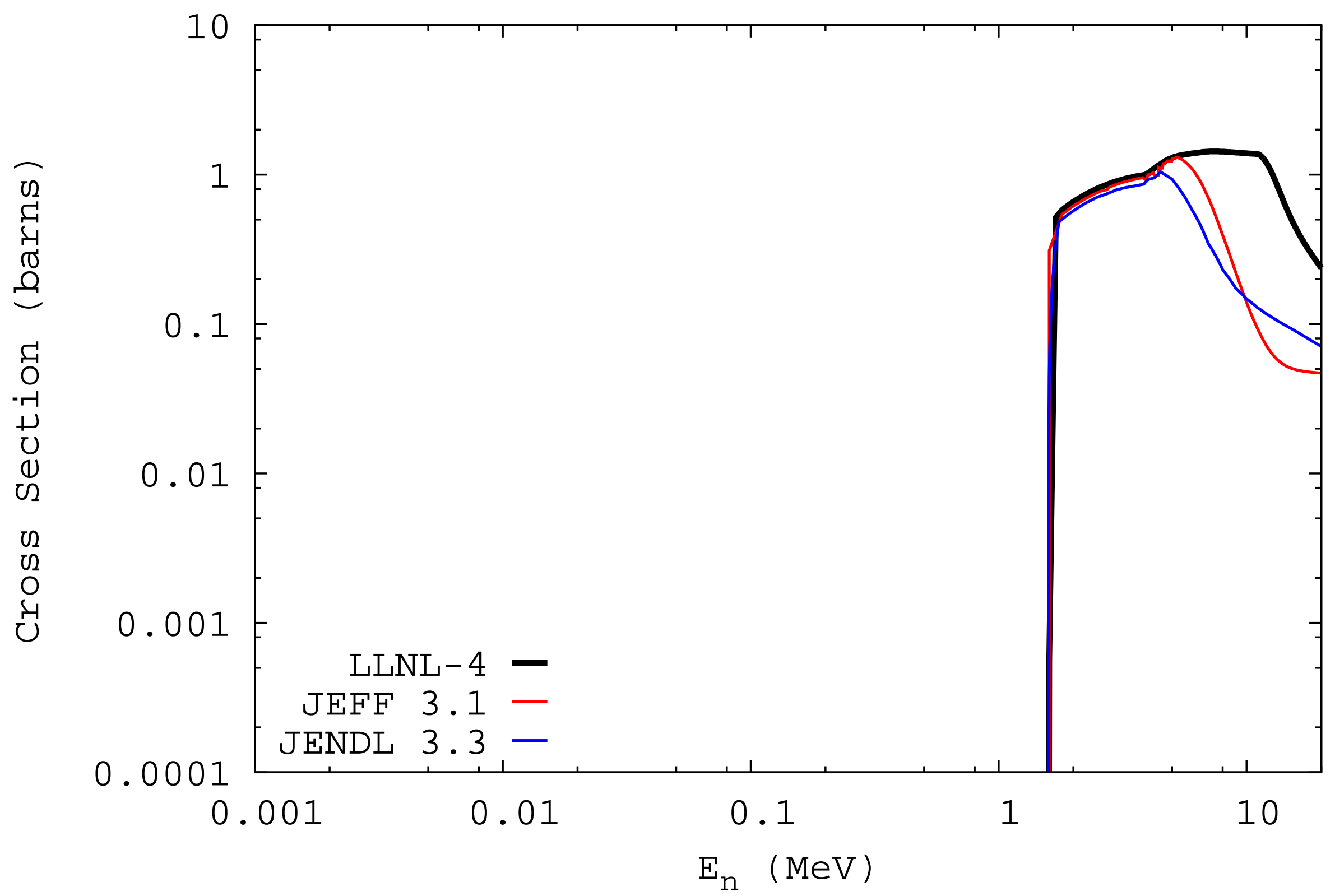


${ }^{50} \mathrm{Ti}_{g}(\mathrm{n}, \mathrm{np})+(\mathrm{n}, \mathrm{pn}){ }^{49} \mathrm{SC}$

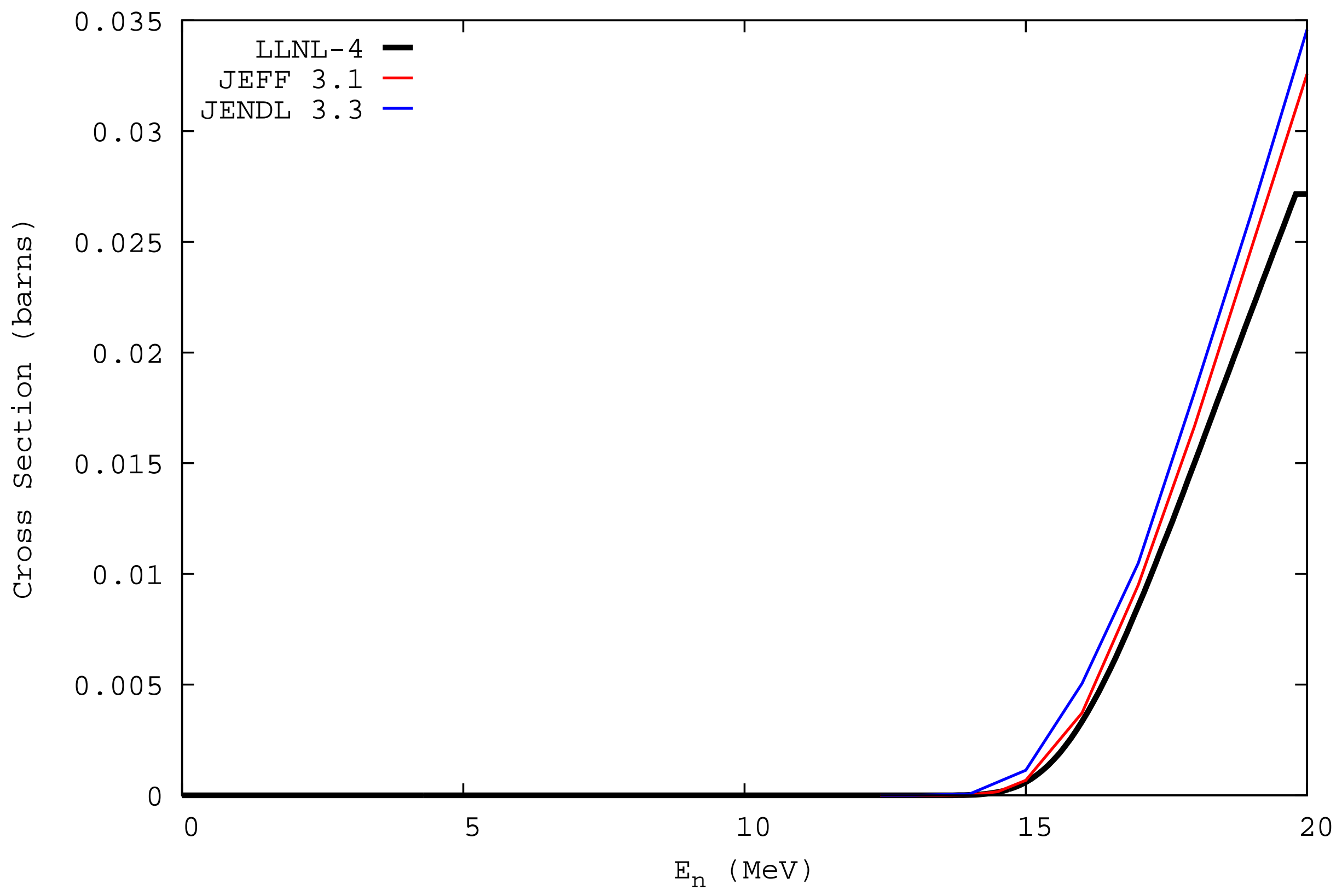


${ }^{50} \mathrm{Ti}_{\mathrm{g}}(\mathrm{n}, \mathrm{p}){ }^{50} \mathrm{SC}$

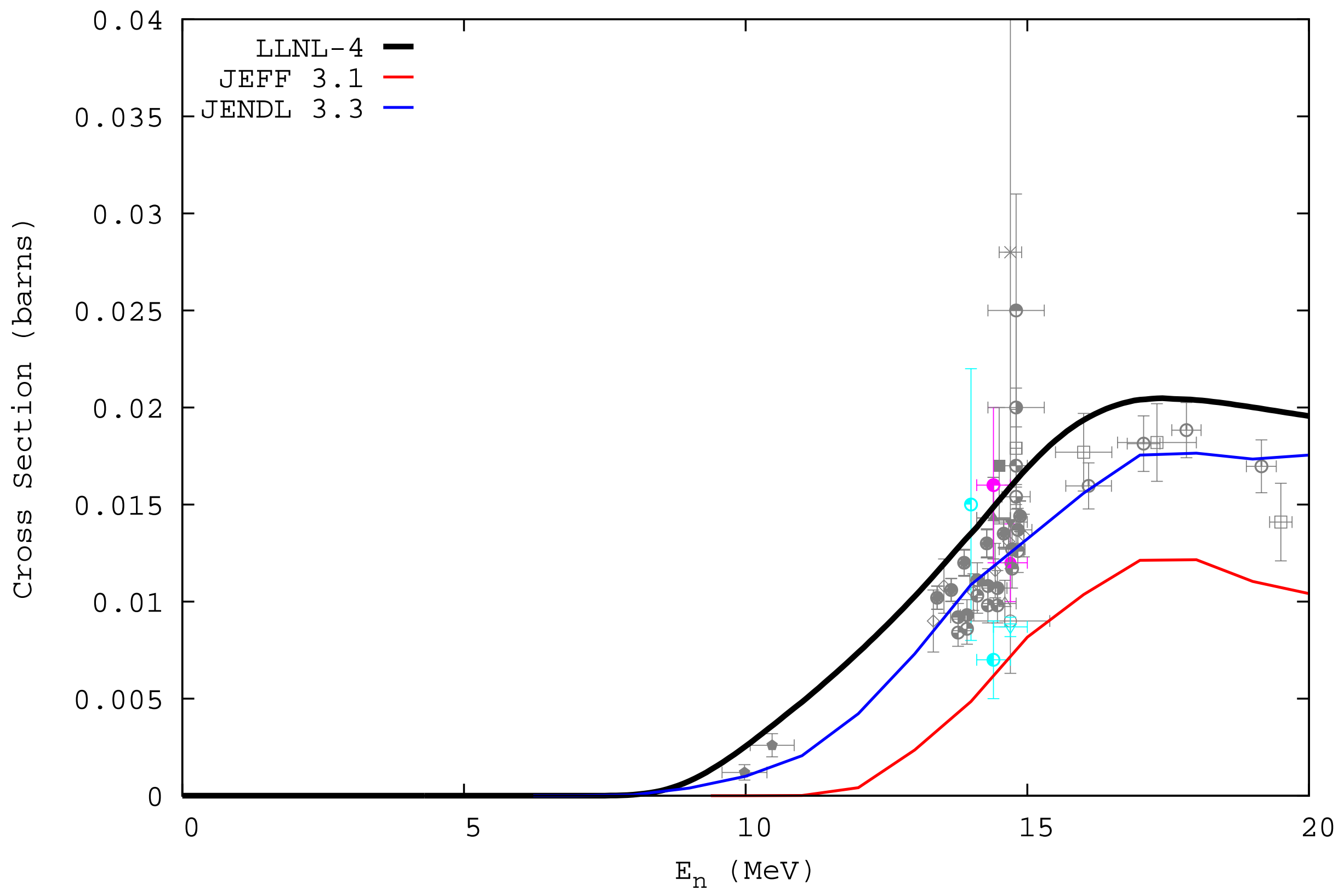




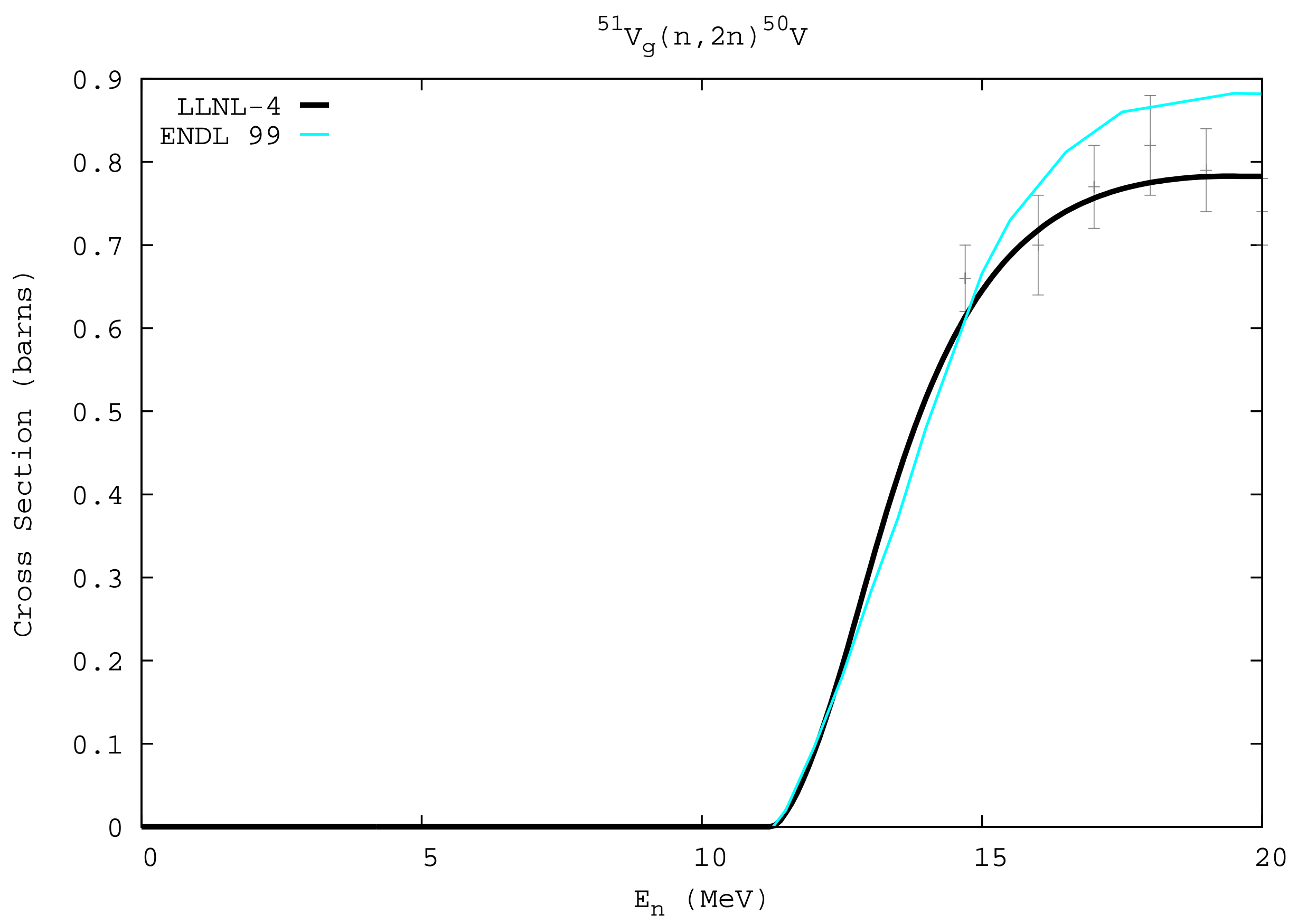


${ }^{51} \mathrm{~V}_{\mathrm{g}}(\mathrm{n}, \alpha){ }^{48} \mathrm{Sc}$

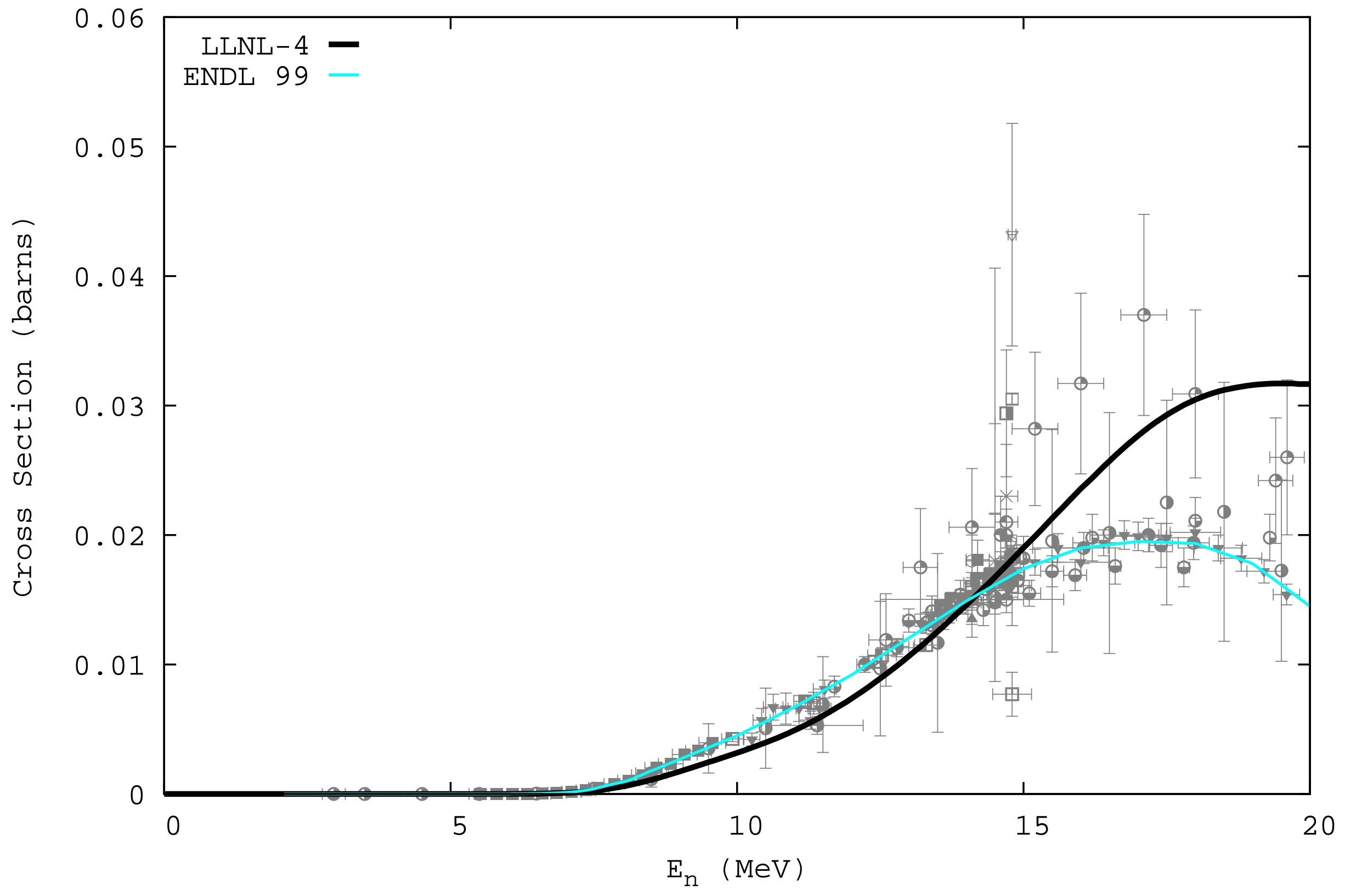




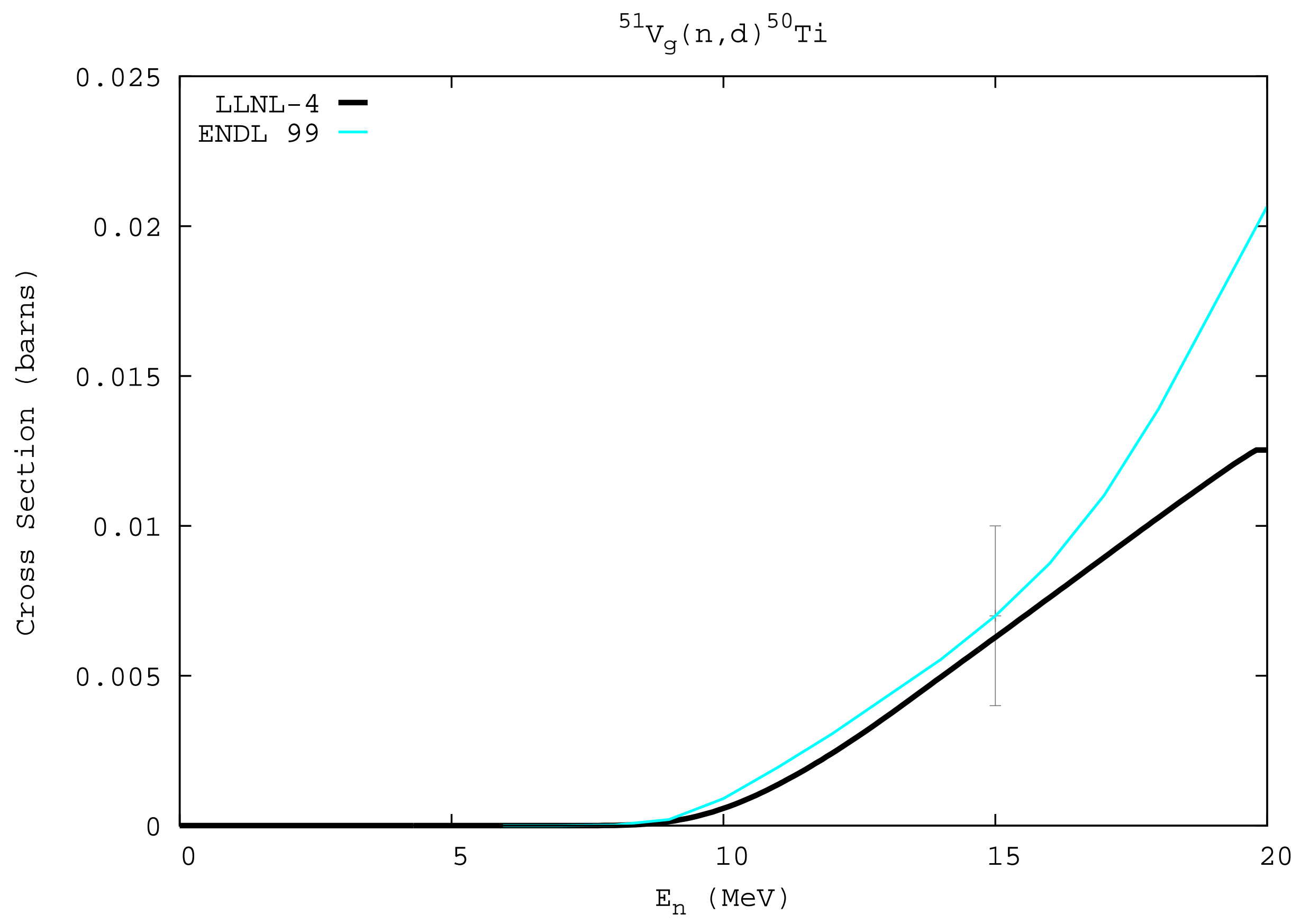




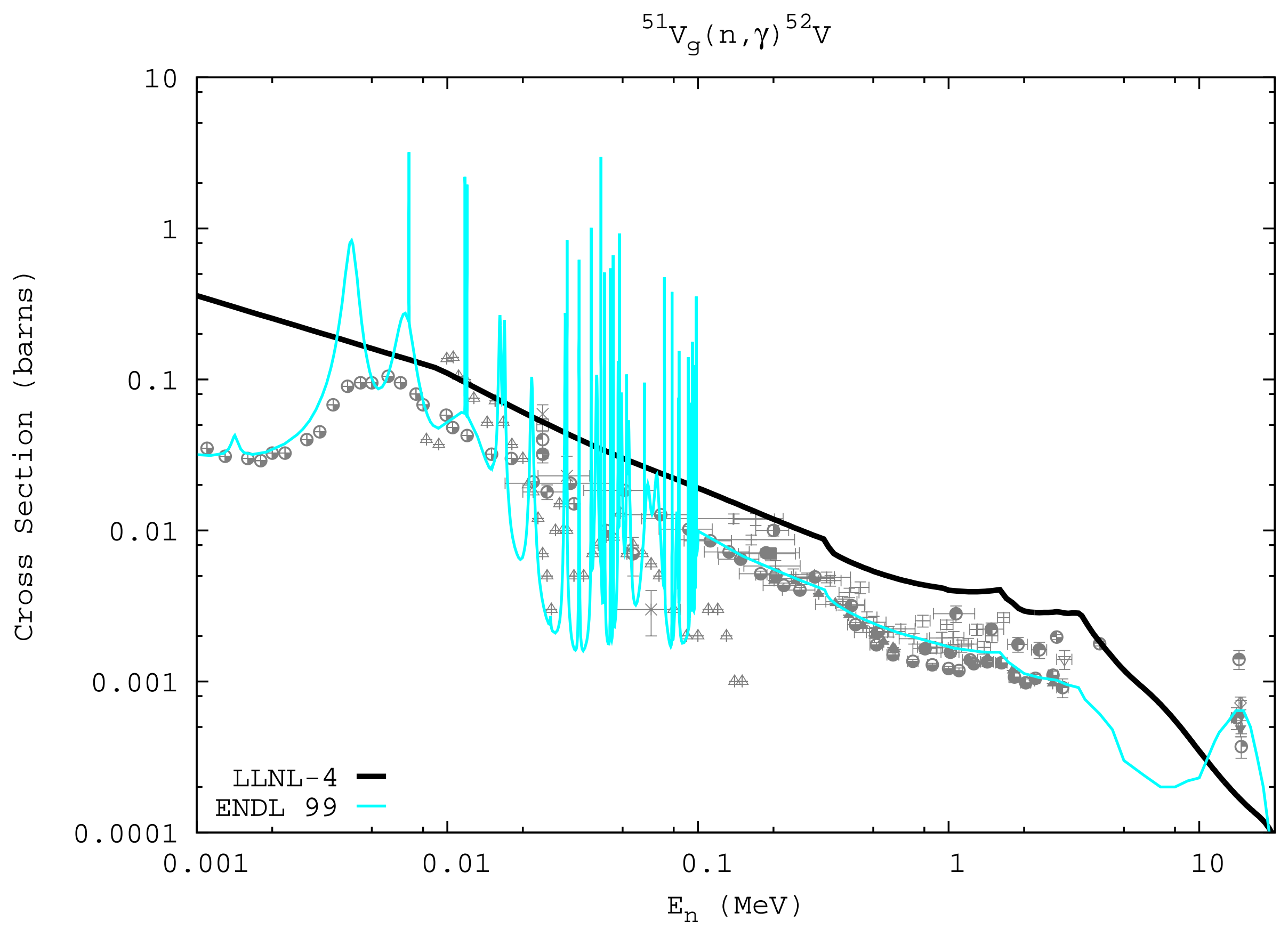




$$
{ }^{51} V_{g}(n, n){ }^{51} V
$$

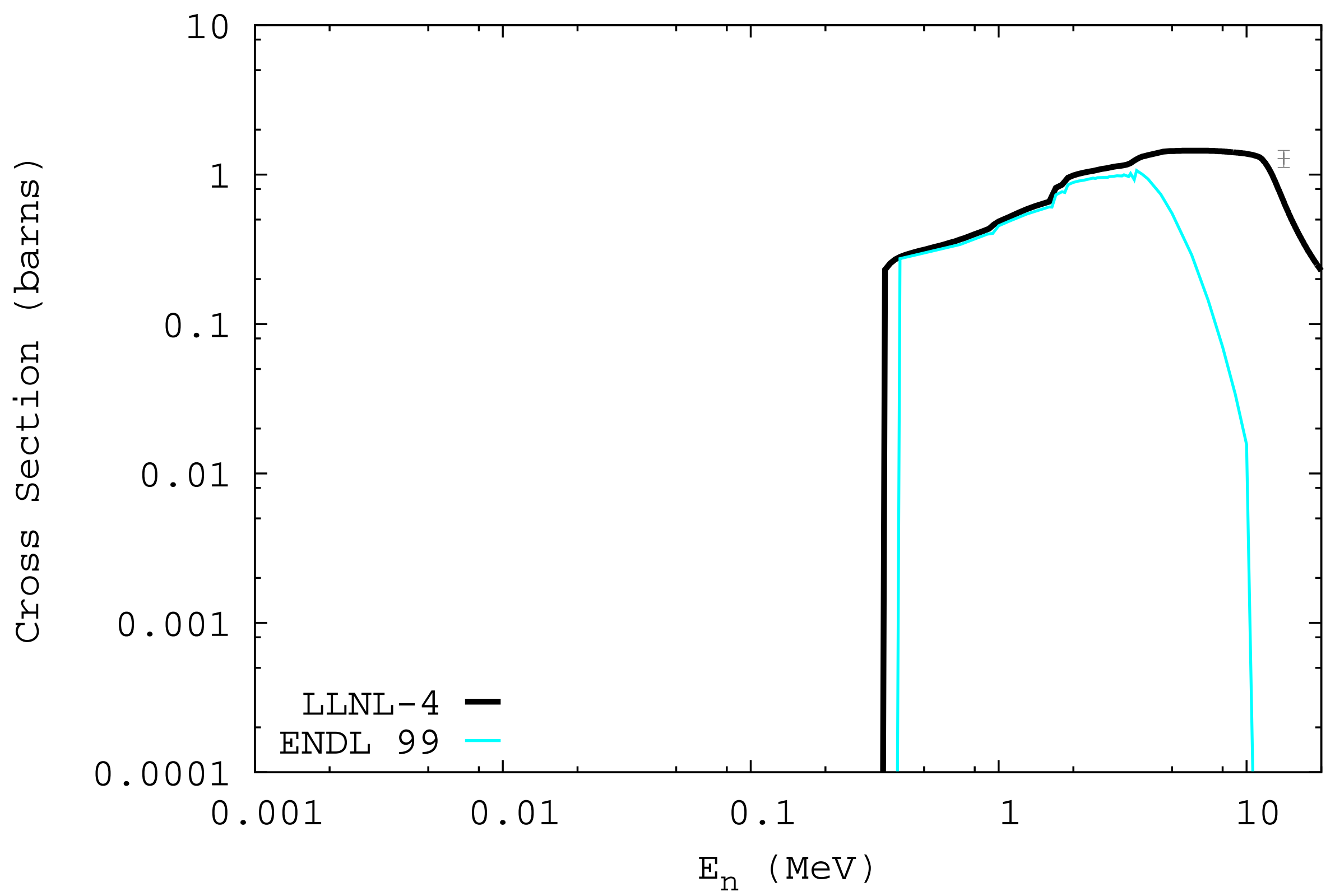




$$
{ }^{51} V_{g}(n, n p)+(n, p n){ }^{50} \mathrm{Ti}
$$

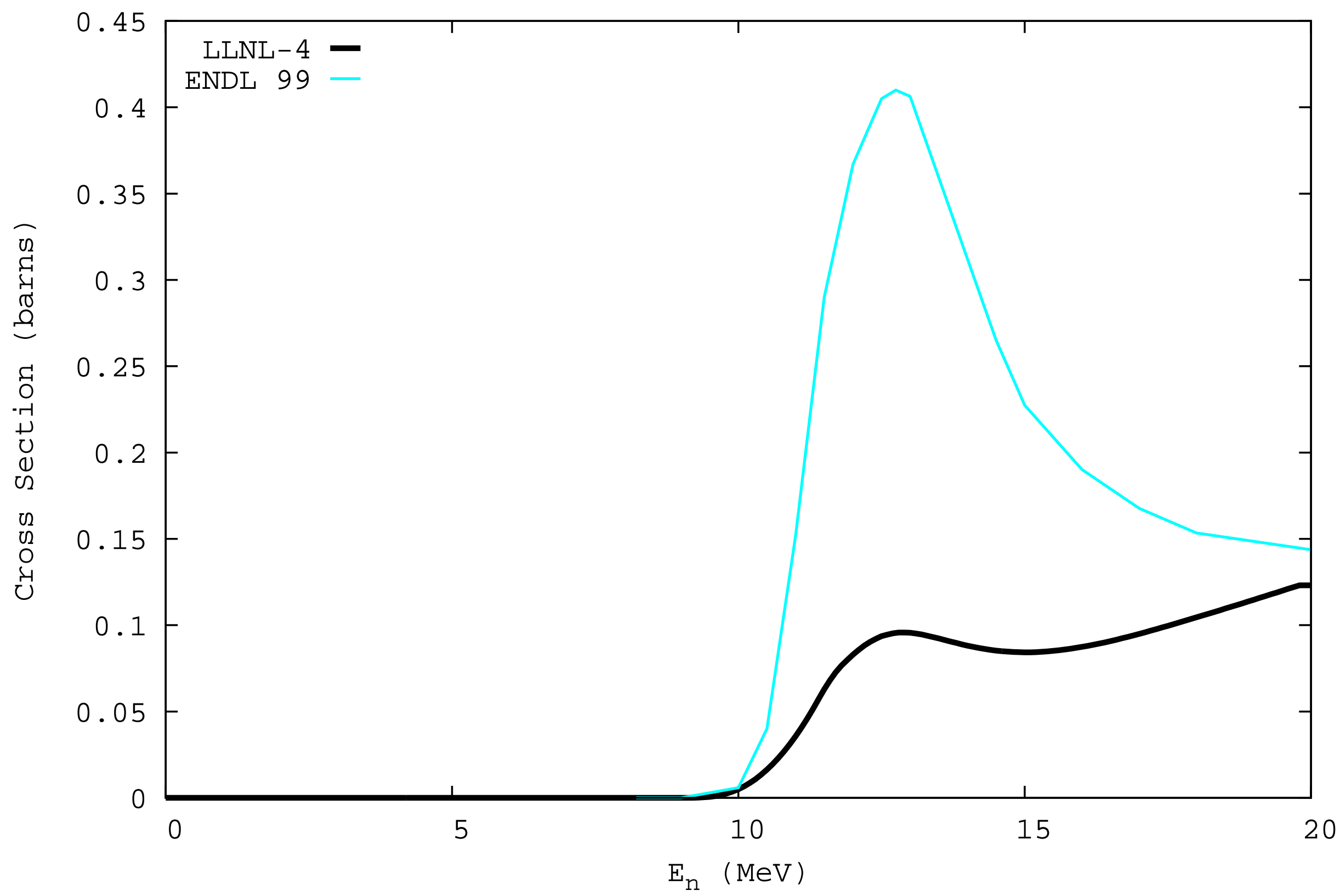




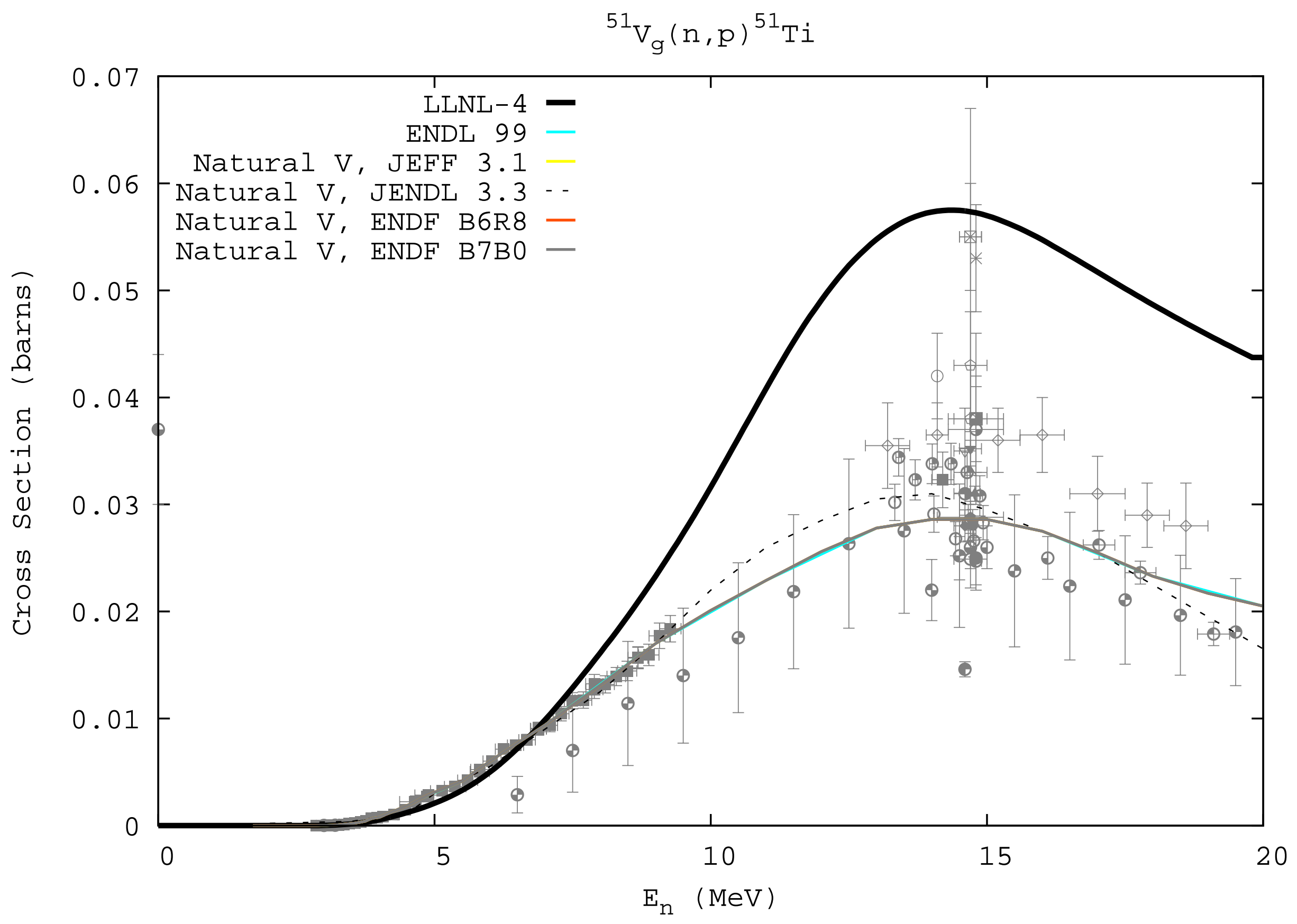

